\title{
Five years of searches for point sources of astrophysical neutrinos with the AMANDA-II neutrino telescope
}

A. Achterberg ${ }^{31}$, M. Ackermann ${ }^{33 *}$, J. Adams ${ }^{11}$, J. Ahrens ${ }^{21}$, K. Andeen ${ }^{20}$, D. W. Atlee ${ }^{29}$, J. N. Bahcall ${ }^{25 \dagger}$, X. Bai ${ }^{23}$, B. Baret ${ }^{9}$, S. W. Barwick ${ }^{16}$, R. Bay $^{5}$, K. Beattie ${ }^{7}$, T. Becka ${ }^{21}$, J. K. Becker ${ }^{13}$, K.-H. Becker ${ }^{32}$, P. Berghaus ${ }^{8}$, D. Berley ${ }^{12}$, E. Bernardini ${ }^{33 *}$, D. Bertrand ${ }^{8}$, D. Z. Besson ${ }^{17}$, E. Blaufuss ${ }^{12}$, D. J. Boersma ${ }^{20}$, C. Bohm ${ }^{27}$, J. Bolmont ${ }^{33}$, S. Böser ${ }^{33}$, O. Botner ${ }^{30}$, A. Bouchta ${ }^{30}$, J. Braun ${ }^{20}$, C. Burgess ${ }^{27}$, T. Burgess ${ }^{27}$, T. Castermans ${ }^{22}$, D. Chirkin ${ }^{7}$, B. Christy $^{12}$, J. Clem $^{23}$, D. F. Cowen ${ }^{29,28}$, M. V. D'Agostino ${ }^{5}$, A. Davour ${ }^{30}$, C. T. Day ${ }^{7}$, C. De Clercq ${ }^{9}$, L. Demirörs ${ }^{23}$, F. Descamps ${ }^{14}$, P. Desiati ${ }^{20}$, T. DeYoung ${ }^{29}$, J. C. Diaz-Velez ${ }^{20}$, J. Dreyer ${ }^{13}$, J. P. Dumm ${ }^{20}$, M. R. Duvoort ${ }^{31}$, W. R. Edwards ${ }^{7}$, R. Ehrlich ${ }^{12}$, J. Eisch $^{26}$, R. W. Ellsworth ${ }^{12}$, P. A. Evenson ${ }^{23}$, O. Fadiran ${ }^{3}$, A. R. Fazely ${ }^{4}$, T. Feser $^{21}$, K. Filimonov ${ }^{5}$, B. D. Fox ${ }^{29}$, T. K. Gaisser ${ }^{23}$, J. Gallagher ${ }^{19}$, R. Ganugapati' ${ }^{20}$, H. Geenen ${ }^{32}$, L. Gerhardt ${ }^{16}$, A. Goldschmidt ${ }^{7}$, J. A. Goodman ${ }^{12}$, R. Gozzini' ${ }^{21}$, S. Grullon ${ }^{20}$, A. Groß $\beta^{15}$, R. M. Gunasingha ${ }^{4}$, M. Gurtner ${ }^{32}$, A. Hallgren ${ }^{30}$, F. Halzen ${ }^{20}$, K. Han ${ }^{11}$, K. Hanson ${ }^{20}$, D. Hardtke ${ }^{5}$, R. Hardtke ${ }^{26}$, T. Harenberg ${ }^{32}$, J. E. Hart ${ }^{29}$, T. Hauschildt ${ }^{23}$, D. Hays ${ }^{7}$, J. Heise ${ }^{31}$, K. Helbing ${ }^{32}$, M. Hellwig ${ }^{21}$, P. Herquet ${ }^{22}$, G. C. Hill20 ${ }^{20}$ J. Hodges ${ }^{20}$, K. D. Hoffman ${ }^{12}$, B. Hommez ${ }^{14}$, K. Hoshina ${ }^{20}$, D. Hubert ${ }^{9}$, B. Hughey ${ }^{20}$, P. O. Hulth ${ }^{27}$, K. Hultqvist ${ }^{27}$, S. Hundertmark ${ }^{27}$, J.-P. Hülß ${ }^{32}$, A. Ishihara ${ }^{10}$, J. Jacobsen ${ }^{7}$, G. S. Japaridze ${ }^{3}$, H. Johansson ${ }^{27}$, A. Jones ${ }^{7}$, J. M. Joseph ${ }^{7}$, K.-H. Kampert ${ }^{32}$, A. Karle ${ }^{20}$, H. Kawai ${ }^{10}$, J. L. Kelley ${ }^{20}$, M. Kestel ${ }^{29}$, N. Kitamura ${ }^{20}$, S. R. Klein ${ }^{7}$, S. Klepser ${ }^{33}$, G. Kohnen ${ }^{22}$, H. Kolanoski ${ }^{6}$, M. Kowalski ${ }^{6}$, L. Köpke ${ }^{21}$, M. Krasberg ${ }^{20}$, K. Kuehn ${ }^{16}$, H. Landsman ${ }^{20}$, H. Leich $^{33}$, D. Leier ${ }^{13}$, M. Leuthold ${ }^{1}$, I. Liubarsky ${ }^{18}$, J. Lundberg ${ }^{30}$,

J. Lünemann ${ }^{13}$, J. Madsen ${ }^{26}$, K. Mase ${ }^{10}$, H. S. Matis ${ }^{7}$, T. McCauley ${ }^{7}$, C. P. McParland ${ }^{7}$, A. Meli ${ }^{13}$, T. Messarius ${ }^{13}$, P. Mészáros ${ }^{29,28}$, H. Miyamoto ${ }^{10}$, A. Mokhtarani ${ }^{7}$, T. Montaruli ${ }^{2 \ddagger}$, A. Morey ${ }^{5}$, R. Morse ${ }^{20}$, S. M. Movit ${ }^{28}$, K. Münich $^{13}$, R. Nahnhauer ${ }^{33}$,

\footnotetext{
* Corresponding authors: markus.ackermann@desy.de (M. Ackermann) and elisa.bernardini@desy.de (E. Bernardini)

$\dagger$ Deceased

$\ddagger$ On leave from University of Bari, I-70126 Bari, Italy
} 
J. W. Nam ${ }^{16}$, P. Nießen ${ }^{23}$, D. R. Nygren ${ }^{7}$, H. Ögelman ${ }^{20}$, A. Olivas ${ }^{12}$, S. Patton ${ }^{7}$, C. Peña-Garay ${ }^{25}$, C. Pérez de los $\operatorname{Heros}^{30}$, A. Piegsa ${ }^{21}$, D. Pieloth ${ }^{33}$, A. C. Pohl ${ }^{30}$, R. Porrata ${ }^{5}$, J. Pretz ${ }^{12}$, P. B. Price ${ }^{5}$, G. T. Przybylski ${ }^{7}$, K. Rawlins ${ }^{2}$, S. Razzaque ${ }^{29,28}$,

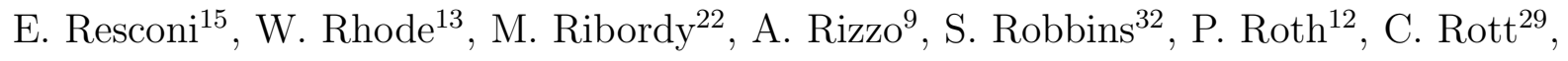
D. Rutledge ${ }^{29}$, D. Ryckbosch ${ }^{14}$, H.-G. Sander ${ }^{21}$, S. Sarkar ${ }^{24}$, S. Schlenstedt ${ }^{33}$, T. Schmidt ${ }^{12}$, D.Schneider ${ }^{20}$, D. Seckel ${ }^{23}$, S. H. Seo ${ }^{29}$, S. Seunarine ${ }^{11}$, A. Silvestri ${ }^{16}$, A. J. Smith ${ }^{12}$, M. Solarz ${ }^{5}$, C. Song ${ }^{20}$, J. E. Sopher ${ }^{7}$, G. M. Spiczak ${ }^{26}$, C. Spiering ${ }^{33}$, M. Stamatikos ${ }^{20}$, T. Stanev ${ }^{23}$, P. Steffen ${ }^{33}$, T. Stezelberger ${ }^{7}$, R. G. Stokstad ${ }^{7}$, M. C. Stoufer ${ }^{7}$, S. Stoyanov ${ }^{23}$, E. A. Strahler ${ }^{20}$, T. Straszheim ${ }^{12}$, K.-H. Sulanke ${ }^{33}$, G. W. Sullivan ${ }^{12}$, T. J. Sumner ${ }^{18}$,

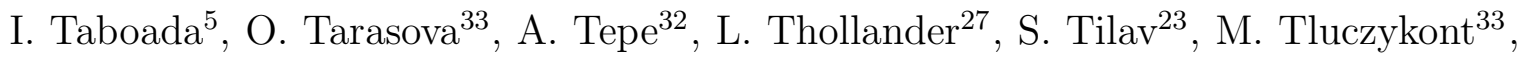
P. A. Toale ${ }^{29}$, D. Turčan ${ }^{12}$, N. van Eijndhoven ${ }^{31}$, J. Vandenbroucke ${ }^{5}$, A. Van Overloop ${ }^{14}$, B. Voigt ${ }^{33}$, W. Wagner ${ }^{29}$, C. Walck ${ }^{27}$, H. Waldmann ${ }^{33}$, M. Walter ${ }^{33}$, Y.-R. Wang ${ }^{20}$, C. Wendt ${ }^{20}$, C. H. Wiebusch ${ }^{1}$, G. Wikström ${ }^{27}$, D. R. Williams ${ }^{29}$, R. Wischnewski ${ }^{33}$, H. Wissing ${ }^{1}$, K. Woschnagg ${ }^{5}$, X. W. Xu ${ }^{26}$, G. Yodh ${ }^{16}$, S. Yoshida ${ }^{10}$, J. D. Zornoza ${ }^{20}$

${ }^{1}$ III Physikalisches Institut, RWTH Aachen University, D-52056, Aachen, Germany

${ }^{2}$ Dept. of Physics and Astronomy, University of Alaska Anchorage, 3211 Providence Dr., Anchorage, AK 99508, USA ${ }^{3}$ CTSPS, Clark-Atlanta University, Atlanta, GA 30314, USA

${ }^{4}$ Dept. of Physics, Southern University, Baton Rouge, LA 70813, USA

${ }^{5}$ Dept. of Physics, University of California, Berkeley, CA 94720, USA

${ }^{6}$ Institut für Physik, Humboldt Universität zu Berlin, D-12489 Berlin, Germany

${ }^{7}$ Lawrence Berkeley National Laboratory, Berkeley, CA 94720, USA

${ }^{8}$ Université Libre de Bruxelles, Science Faculty CP230, B-1050 Brussels, Belgium

${ }^{9}$ Vrije Universiteit Brussel, Dienst ELEM, B-1050 Brussels, Belgium

${ }^{10}$ Dept. of Physics, Chiba University, Chiba 263-8522 Japan

${ }^{11}$ Dept. of Physics and Astronomy, University of Canterbury, Private Bag 4800, Christchurch, New Zealand

${ }^{12}$ Dept. of Physics, University of Maryland, College Park, MD 20742, USA

${ }^{13}$ Dept. of Physics, Universität Dortmund, D-44221 Dortmund, Germany

${ }^{14}$ Dept. of Subatomic and Radiation Physics, 


\author{
University of Gent, B-9000 Gent, Belgium \\ ${ }^{15}$ Max-Planck-Institut für Kernphysik, D-69177 Heidelberg, Germany \\ ${ }^{16}$ Dept. of Physics and Astronomy, University of California, Irvine, CA 92697, USA \\ ${ }^{17}$ Dept. of Physics and Astronomy, University of Kansas, Lawrence, KS 66045, USA \\ ${ }^{18}$ Blackett Laboratory, Imperial College, London SW7 2BW, UK \\ ${ }^{19}$ Dept. of Astronomy, University of Wisconsin, Madison, WI 53706, USA \\ ${ }^{20}$ Dept. of Physics, University of Wisconsin, Madison, WI 53706, USA \\ ${ }^{21}$ Institute of Physics, University of Mainz, \\ Staudinger Weg 7, D-55099 Mainz, Germany \\ ${ }^{22}$ University of Mons-Hainaut, 7000 Mons, Belgium \\ ${ }^{23}$ Bartol Research Institute, University of Delaware, Newark, DE 19716, USA \\ ${ }^{24}$ Dept. of Physics, University of Oxford, \\ 1 Keble Road, Oxford OX1 3NP, UK \\ ${ }^{25}$ Institute for Advanced Study, Princeton, NJ 08540, USA \\ ${ }^{26}$ Dept. of Physics, University of Wisconsin, River Falls, WI 54022, USA \\ ${ }^{27}$ Dept. of Physics, Stockholm University, SE-10691 Stockholm, Sweden \\ ${ }^{28}$ Dept. of Astronomy and Astrophysics, \\ Pennsylvania State University, University Park, PA 16802, USA \\ ${ }^{29}$ Dept. of Physics, Pennsylvania State University, University Park, PA 16802, USA \\ ${ }^{30}$ Division of High Energy Physics, Uppsala University, S-75121 Uppsala, Sweden \\ ${ }^{31}$ Dept. of Physics and Astronomy, Utrecht University/SRON, \\ NL-3584 CC Utrecht, The Netherlands \\ ${ }^{32}$ Dept. of Physics, University of Wuppertal, D-42119 Wuppertal, Germany and \\ ${ }^{33}$ DESY, D-15735 Zeuthen, Germany
}

(Dated: May 1, 2007)

We report the results of a five-year survey of the northern sky to search for point sources of high energy neutrinos. The search was performed on the data collected with the AMANDA-II neutrino telescope in the years 2000 to 2004, with a livetime of 1001 days. The sample of selected events consists of 4282 upward going muon tracks with high reconstruction quality and an energy larger than about 100 
$\mathrm{GeV}$. We found no indication of point sources of neutrinos and set $90 \%$ confidence level flux upper limits for an all-sky search and also for a catalog of 32 selected sources. For the all-sky search, our average (over declination and right ascension) experimentally observed upper limit $\Phi^{0}=\left(\frac{\mathrm{E}}{1 \mathrm{TeV}}\right)^{\gamma} \cdot \frac{\mathrm{d} \Phi}{\mathrm{dE}}$ to a point source flux of muon and tau neutrino (detected as muons arising from taus) is $\Phi_{\nu_{\mu}+\bar{\nu}_{\mu}}^{0}+\Phi_{\nu_{\tau}+\bar{\nu}_{\tau}}^{0}=$ $11.1 \cdot 10^{-11} \mathrm{TeV}^{-1} \mathrm{~cm}^{-2} \mathrm{~s}^{-1}$, in the energy range between $1.6 \mathrm{TeV}$ and $2.5 \mathrm{PeV}$ for a flavor ratio $\Phi_{\nu_{\mu}+\bar{\nu}_{\mu}}^{0} / \Phi_{\nu_{\tau}+\bar{\nu}_{\tau}}^{0}=1$ and assuming a spectral index $\gamma=2$. It should be noticed that this is the first time we set upper limits to the flux of muon and tau neutrinos. In previous papers we provided muon neutrino upper limits only neglecting the sensitivity to a signal from tau neutrinos, which improves the limits by $10 \%$ to $16 \%$. The value of the average upper limit presented in this work corresponds to twice the limit on the muon neutrino flux $\Phi_{\nu_{\mu}+\bar{\nu}_{\mu}}^{0}=5.5 \cdot 10^{-11} \mathrm{TeV}^{-1} \mathrm{~cm}^{-2} \mathrm{~s}^{-1}$. A stacking analysis for preselected active galactic nuclei and a search based on the angular separation of the events were also performed. We report the most stringent flux upper limits to date, including the results of a detailed assessment of systematic uncertainties.

PACS numbers: 95.55.Vj, 95.75.Mn, 95.75.Pq, 95.80.+p, 95.85.Ry

\section{INTRODUCTION}

The search for high energy extraterrestrial neutrinos is the major focus of the Antarctic Muon And Neutrino Detector Array (AMANDA) [1]. The goal is the understanding of the origin of high energy cosmic rays. While a flux of charged particles is observed up to energies of a few hundred EeV, high energy gamma rays with energies up to a few tens TeV have been detected from several astrophysical objects. Remarkably, the nature of the high energy processes leading to the observed particles and radiation is in most cases not known.

Neutrinos are expected to be emitted from a variety of astrophysical objects: galactic objects like pulsars [2], accreting binary systems [3], particularly micro-quasars [4, 5, 6], and supernova remnants [7], as well as from extragalactic objects like active galactic nuclei [8, 9], particularly blazars [10, 11, 12, 13]. Reviews that include flux predictions of high energy neutrinos from galactic and extragalactic objects can be found in [7] and [14]. To date no 
extraterrestrial high energy neutrino flux has been observed [15, 16, 17, 18, 19]. Searches for point sources of high energy neutrinos were presented by [20, 21, 22, 23, 24].

The search for cosmic neutrinos appears more challenging than the observation of cosmic rays and high energy gamma-rays, due to the much smaller cross section for neutrino interaction. On the other hand, the small interaction cross section makes neutrinos rather unique astronomical messengers: neutrinos point back to their origin and unlike gamma-rays they can escape from dense matter regions and propagate freely over cosmological distances. Their observation would provide an incontrovertible signature of hadron acceleration by astrophysical objects.

In this paper we report the results of a search for point sources of high energy neutrinos using data collected with AMANDA-II between 2000 and 2004.

\section{DETECTION OF UPWARD GOING NEUTRINOS WITH AMANDA}

The AMANDA-II detector is located at the Geographic South Pole and consists of an array of photomultipliers to detect Cherenkov photons emitted by charged particles traversing the polar ice. An individual detection unit (optical module) is assembled from an 8 inch diameter photomultiplier, providing good sensitivity to single photons, housed in a pressure-resistant glass sphere, both optically coupled with transparent gel. The system has been mechanically and optically stable since the first year of deployment (1996). Completed in the year 2000, the detector includes 677 optical modules on 19 vertical strings, most of which are deployed at depths between 1.5 and 2 kilometers [1]. Approximately 540 of the optical modules that form the core of the detector array and showing stable performance are used for this analysis.

The geometry of AMANDA-II is optimized to detect muon tracks induced by charged current interactions of neutrinos with energies above $1 \mathrm{TeV}$. Neutrino induced muon tracks may have ranges of several kilometers (about $8 \mathrm{~km}$ in ice at $10 \mathrm{TeV}$ ). They are reconstructed from the arrival time of the Cherenkov photons at the optical modules. The energy threshold depends on reconstruction methods and quality criteria. In this analysis $95 \%$ of the Monte Carlo simulated atmospheric neutrinos have energies larger than about $100 \mathrm{GeV}([25$, 26] ). The muon energy can be estimated from the number of detected Cherenkov photons. The resolution in the logarithm of the energy, $\log _{10}(\mathrm{E} / \mathrm{GeV})$, is about 0.4 at energies above a 
few $\mathrm{TeV}$ [27]. Above $1 \mathrm{TeV}$, the mean angular offset between the incoming neutrino and the muon track is less than $0.8^{\circ}[28]$. The mean scattering angle due to multiple Coulombscattering during propagation of the muon is an order of magnitude smaller [29].

Searches for astrophysical sources of neutrinos have to cope with a background of events from the interaction of cosmic rays in the earth's atmosphere. Decays of secondary mesons induce a background of downward going muons and a more uniform background of neutrinos. Typical trigger rates measured with AMANDA-II are $O\left(10^{9}\right)$ events per year from downward going atmospheric muons and $O\left(10^{3}\right)$ muon tracks induced by atmospheric neutrinos, while only a few events are predicted by models for astrophysical sources [30, 31]. Neutrino candidates are selected by rejecting muon tracks reconstructed as downward going since only neutrinos can cross the earth. This limits the sensitivity to the northern sky.

A point source would manifest itself as a localized excess of events over the background. While the background is uniformly distributed in right ascension, the angular distribution of an astrophysical signal would follow the detector point spread function. In order to achieve a high signal-to-noise ratio, much effort was dedicated to improving the event reconstruction and selection, and consequently the track angular resolution, over a wide energy range.

A series of reconstruction methods with increasing accuracy at the expense of increased reconstruction time are applied. Fast pattern recognition procedures provide a first-guess estimate of the track direction. Because of the scattering of the photons on dust and crystal grains in the polar ice [32], complex reconstruction algorithms are necessary to measure the direction with a good angular resolution. Based on maximum likelihood procedures in a multi-parameter space using the first-guess results as starting point, high level reconstructions aim at finding the best likelihood for a given event topology with respect to the recorded hits [27].

About $0.1 \%$ of the downward going muons are wrongly reconstructed as upward going. A selection based on event quality parameters is used to reduce these events by an additional four orders of magnitude. Yet an irreducible background remains from upward going muons induced by atmospheric neutrinos together with a small fraction of mis-reconstructed downward going muons plus possible signal events. Typical resolutions achieved in the reconstruction of the muon direction are between $1.5^{\circ}$ and $2.5^{\circ}$ degrees (median spatial angle), depending on energy and declination.

In order to avoid biases in the event selection, the final event selection was developed 
following a blind approach. In the search for point sources, where the event direction is used to look for a signal, this is accomplished by optimizing cuts on a sample of events with randomized right ascension. Accumulations of events due to signal would be averaged out, while the dependency of the detection efficiency on declination is preserved. The background is estimated from the detected events, by adopting a technique similar to the "off-source" method in gamma-ray astronomy. The error of the background estimation is therefore small and statistical only, independent of the detector simulation.

The detection efficiency for astrophysical neutrinos is studied with a complete Monte Carlo description of neutrinos fluxes, propagation through the earth and interactions, of the muon propagation and of the detector response [33]. The latter takes into account the propagation of photons in the ice and the photon detection probability. The systematic uncertainties in this modeling affect the signal efficiency and therefore the calculation of flux upper limits or, in case of detection, the precision with which the cosmic neutrino flux can be measured. Comparison of the final event sample to the Monte Carlo expectation for atmospheric neutrinos allows the verification of the modeling accuracy and of the detection efficiency. These aspects will be addressed in detail in Section $\mathrm{V}$.

\section{EVENT RECONSTRUCTION AND SELECTION}

The searches reported in this paper use the data collected with the AMANDA-II detector in the years 2000 to 2004. The austral-summer data (from November to February), taken during the detector maintenance and station summer activity periods, are excluded. Periods of overall detector instability are also discarded. The remaining live-time is 1001 days, after correction for the intrinsic DAQ dead-time. The trigger used to collect this data requires at least 24 optical modules (OM) recording one or more pulses above threshold (hits) within $2.5 \mu \mathrm{s}$.

Table [1] shows the first three filtering levels used to process the $8.9 \times 10^{9}$ events used in this analysis. The multi-level filtering is needed because the final reconstruction algorithms are too CPU-intensive to use on the entire dataset. Sophistication and CPU demand per event of these procedures increase with level, as does the tightness of cuts for background rejection. The event passing rates in Table $\square$ are normalized to the number of triggered events $\left(8.9 \times 10^{9}\right)$. Level 1 and Level 2 of the event reconstruction and selection are based 
on relatively loose cuts, in order to extract an event sample which is still useful for other analyses.

Details of the pre-processing techniques (hit and optical module selection) and of the reconstruction algorithms can be found in [27]. Before reconstruction, short pulses are removed which can be ascribed to electronic noise. Hits from unstable optical modules are also rejected based on their typical TDC and dark noise rates compared to the average (hit and optical module selection in Table I). Events are required to have at least 24 modules hit after this cleaning as in the hardware trigger (re-trigger in Table I) .

Two fast pattern recognition algorithms are then applied to reconstruct the direction of the muons: DirectWalk, described in [27], and JAMS. JAMS provides an enhanced downward going muon track rejection power compared to previous results [34, 35]. The best guess for the direction of a muon track is found from the distribution of hits projected on a plane orthogonal to a candidate track direction. Only hits with a short delay compared to the arrival time expected for the direction of the track hypothesis are considered. Photons generating such "direct" hits have undergone only a few scatters in the ice and have therefore preserved the directional information. The track direction hypothesis is then varied and the distribution of the hit projections studied. The direction with the largest and most isotropic cluster of associated hits is chosen as JAMS result.

\begin{tabular}{c|l|l|l|c} 
Level & Hit/Event filter & Track reconstruction & Event cut & Events kept \\
\hline 1 & Hit \& OM & & hit multiplicity $>23$ & $95.0 \%$ \\
& Re-trigger & & $\theta_{\text {DW }}>70^{\circ}$ & $3.7 \%$ \\
\hline 2 & & DirectWalk & $\theta_{\text {JAMS }}>80^{\circ}$ & $0.4 \%$ \\
& Cross-talk & JAMS & & \\
\hline 3 & & Unbiased likelihood fit (UL) & $\theta_{\mathrm{UL}}>80^{\circ}$ & $0.1 \%$ \\
& & Bayesian likelihood fit (BL) & &
\end{tabular}

TABLE I: Summary of the reconstruction and filtering steps as explained in the text for the first three levels of data reduction, with the fraction of events passing each level compared to the number of triggered events $\left(8.9 \times 10^{9}\right)$.

With JAMS we are able to reject classes of downward going muons which the DirectWalk fit wrongly reconstructs as upward going particles. As a consequence, an efficient reduction of the background from atmospheric muons by a factor of 250 can be achieved at Level 2 of 
the event selection, applying angular cuts to the directions from both first-guess algorithms (cfr. Table I). A filter based on the amplitude and duration of hits and on a talker-receiver map is then applied to exclude pulses induced along twisted-pair cables when analog signals are transmitted from optical modules to the surface (cross-talk in Table I).

Two iterative reconstructions follow: an unbiased likelihood fit (UL), seeded with the result of JAMS and with 32 randomly chosen input directions, and a Bayesian likelihood fit (BL), seeded with the results of UL and with 64 randomly chosen input directions. The Bayesian fit incorporates a prior hypothesis with a parameterization of the MC zenith distribution for atmospheric muons at the detector [27]. The final direction is defined by the best likelihood found.

At Level 3 the data sample is reduced to $9.9 \times 10^{6}$ tracks and is still dominated by downward going muons, outnumbering neutrinos by three orders of magnitude. Fake events due to non-simulated electronic artifacts are rejected after Level 3 with a filter sensitive to correlated noise [36]. The event reconstruction and selection has proved to be stable with respect to these detector instabilities.

Neutrino induced upward going tracks are selected after Level 3 by imposing event quality requirements based on the single track angular resolution and on topological parameters describing the distribution of hits along the trajectories. Three independent parameters are chosen: a) the event based angular resolution, proportional to the width of the likelihood minimum and derived from the fit error matrix [37], b) the smoothness, a parameter describing the homogeneity of the hits along the track [27], and c) the ratio of the likelihoods from the unbiased and the Bayesian reconstructions.

Distributions of these observables were constructed for both data and signal Monte Carlo in 22 declination bands. Together with the search bin radius of the binned search defined in Section VI, the parameter space of these variables is scanned to find the optimum selection with respect to signal efficiency and residual background. The optimum selection provides the best sensitivity as the average upper limit in absence of a signal [38, 39].

The optimum selection criteria determined with this method depend on the assumed signal light deposited and therefore on the assumed signal energy spectrum and on the track direction. We implemented event cut optimizations assuming different signal energy powerlaw spectra $\frac{d \Phi}{d E}=\Phi^{0} \cdot(E / 1 \mathrm{TeV})^{-\gamma}$, with $\Phi^{0}$ as normalization. Two spectral indices were considered: $\gamma=2$, generally assumed to be the most likely for astrophysical beam dumps, 


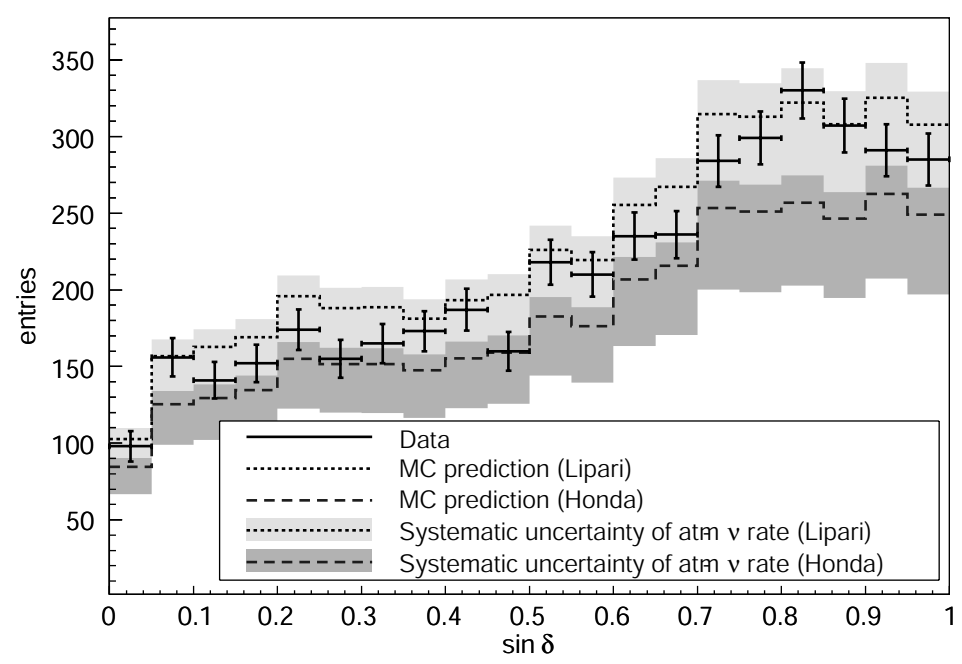

FIG. 1: Declination angle distribution of the final event selection compared to the expectation from Monte Carlo simulation of atmospheric neutrinos, including the systematic error band (see Section $(\mathrm{V})$. The two extremes [25, 26] among different predictions are shown. Error bars on the data point are statistical.

following Fermi shock acceleration of protons, and $\gamma=3$ as a possible extreme of softer spectrum scenarios ${ }^{1}$. We chose cut values which are close to the individual optima and provide a good sensitivity in both cases.

The optimum size of the circular search bins varies between $2.25^{\circ}$ and $3.75^{\circ}$ depending on declination. These search bins contain $60 \%$ to $80 \%$ of the simulated signal, respectively.

\section{PROPERTIES OF THE FINAL EVENT SAMPLE}

A final sample of 4282 upward going muon-like events survived the cuts. This is in agreement with expectations from a Monte Carlo simulation of atmospheric neutrinos following the parametrization in [25]. The central value of this parametrization yields $4600_{-1000}^{+300}$ (sys) expected events. The systematic error is discussed in Section $\mathrm{V}$.

We estimate the contamination from mis-reconstructed downward going events to be less than $5 \%$. This is obtained from the comparison of the event sample after Level 3 of the data reduction to the prediction from atmospheric neutrinos, as a function of the quality of the

\footnotetext{
${ }^{1} \mathrm{~A} \gamma=2$ spectrum with a $1 \mathrm{TeV}$ cutoff was also considered, however the optimum selection found is identical to the $\gamma=3$ case. Therefore this case is omitted here.
} 
reconstructed tracks [40].

Figure 1 compares the observed declination distribution to the one expected for atmospheric neutrinos. Simulation results are given for two different parameterizations of atmospheric neutrino fluxes [25, 26]. The systematic errors are indicated by shadowed areas (see Section $(\mathrm{V})$. The angular distribution confirms that the background for sources other than atmospheric neutrinos is small, within the model uncertainties.

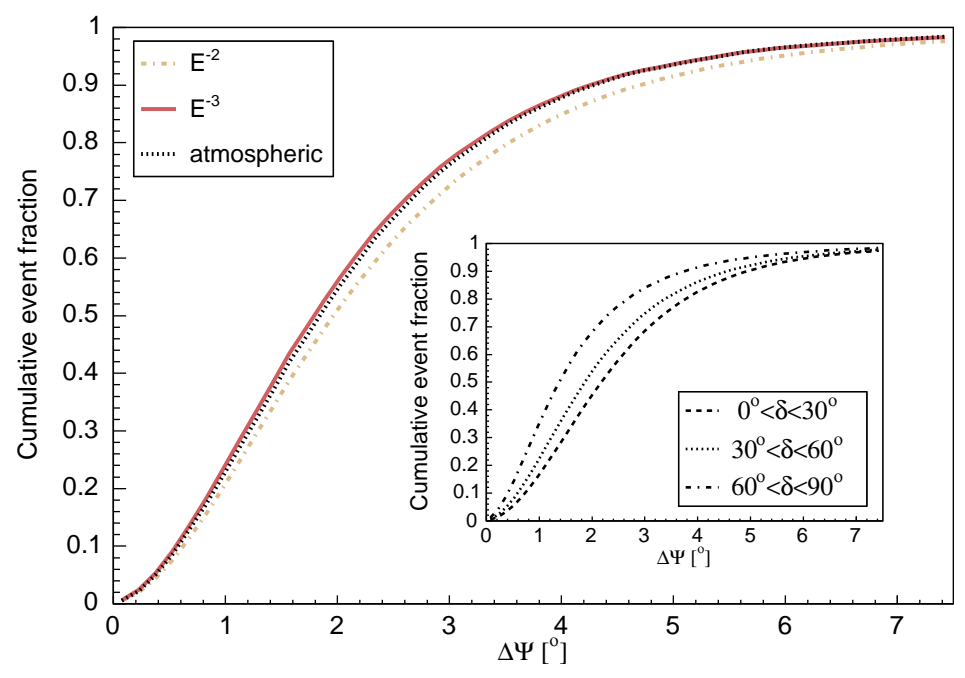

FIG. 2: Cumulative point spread function for the events passing the selection, for different simulated energy spectra. The inset graph shows the dependence on declination, for a spectral index $\gamma=2 . \Delta \Psi$ is the space angle difference between the true and the reconstructed direction of simulated events.

The average pointing resolution of the selected events can be estimated from the distribution of the directional difference between simulated neutrino tracks and reconstructed muon tracks of Monte Carlo events. For a spectral index $\gamma=2$, the median value of the space angle distribution is typically $2^{\circ}$. Figure 2 shows the cumulative point spread function after the final event cuts for different energy spectra. It can be seen that the angular resolution is declination dependent.

The directions of the selected upward going events are shown in Fig. 3. Our event selection yields a relatively uniform coverage of the northern sky. Moreover, the polar location of AMANDA assures an equal exposure for all declinations, independent of the detector operation periods.

This analysis is primarily sensitive to events induced by muon neutrino charged current 


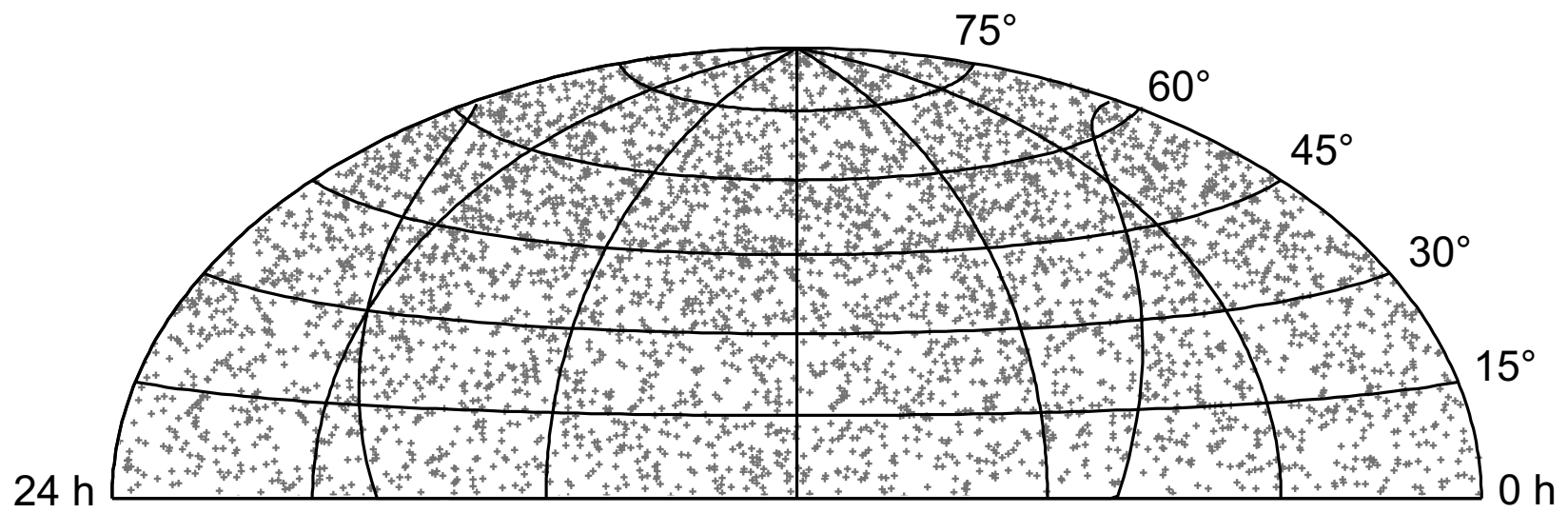

FIG. 3: Sky-plot of the selected 4282 upward going neutrino candidate events. Horizontal coordinates are given as right ascension and vertical coordinates as declination. The black line marks the location of the galactic plane.

interactions. Tau neutrinos give an additional contribution via charged current interactions followed by the $\tau^{ \pm} \rightarrow \mu^{ \pm}+\bar{\nu}_{\mu}\left(\nu_{\mu}\right)+\nu_{\tau}\left(\bar{\nu}_{\tau}\right)$ decay, with a $17.7 \%$ branching ratio [41], which is included in the upper limits reported in Section VI. To estimate the tau neutrino contribution to the final event sample, tau neutrinos were generated according to [28] and propagated through the standard AMANDA-II simulation chain.

Under the assumption of equal fluxes of cosmic muon and tau neutrinos at the earth, the additional contribution of tau neutrino signal events ranges from $10 \%$ to $16 \%$ for $\gamma=2$, depending on declination. This assumption is in accordance with the generally assumed scenario of a flavor ratio at the earth of $\Phi_{\nu_{e}}: \Phi_{\nu_{\mu}}: \Phi_{\nu_{\tau}}=1: 1: 1$, after neutrino oscillation. Deviations from this case can emerge at high energies, where in some astrophysical scenarios the contribution to the neutrino flux from muon decay is suppressed [42], leading to $\Phi_{\nu_{e}}: \Phi_{\nu_{\mu}}: \Phi_{\nu_{\tau}}=1: 1.8: 1.8$. However, equal muon and tau neutrino fluxes are still expected in this scenario.

Two-flavor oscillations of atmospheric neutrinos were simulated with $\Delta m_{2,3}{ }^{2}=2.5 \times$ $10^{-3} \mathrm{eV}^{2}$ and maximum mixing $\theta_{2,3}=45^{\circ}$ [43]. For our sensitive energy range, this results in a disappearance of muon neutrinos of less than $3 \%$, depending on the direction. The corresponding appearance of tau neutrinos leads to an increase of the detected muon rate which is less than $0.5 \%$ and is neglected for this analysis.

The neutrino effective area is a convolution of the neutrino interaction cross section, the muon survival probability and the detector response (geometry and detection efficiencies). 
It depends on the neutrino energy and direction as shown in Figure 4 for muon neutrinos (left) and tau neutrinos (right), including the earth shadowing effect.
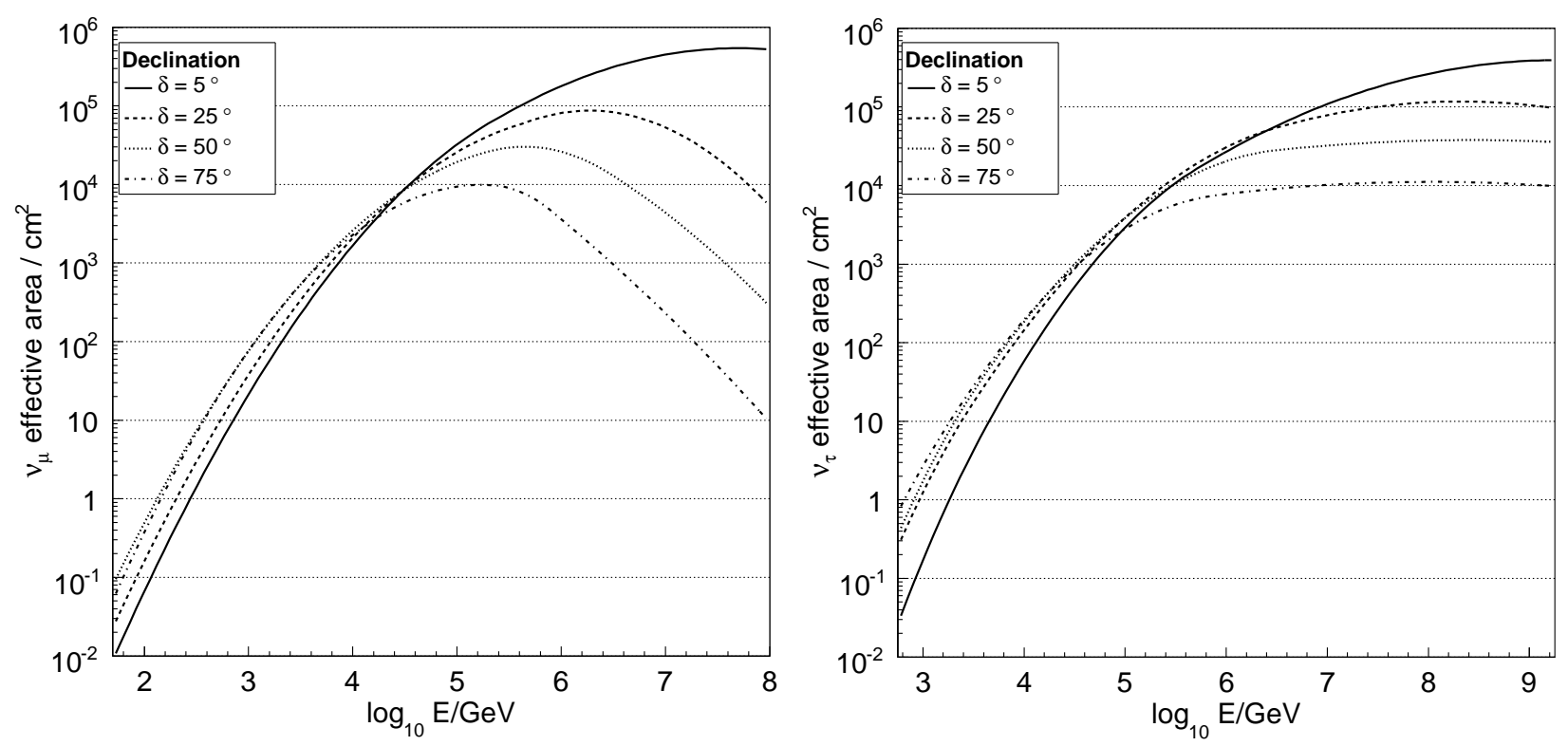

FIG. 4: Neutrino effective area as a function of the neutrino energy for different declinations for muon neutrinos (left) and tau neutrinos (right) respectively. The decrease observed at high energies on the left is due to neutrino absorption in the earth.

Because the event selection optimization allows wider spectral scenarios than in our previous point source searches [34, 35], the sample of selected up-going events contains a significant contribution from low energy events $\left(E_{\nu}<1 \mathrm{TeV}\right)$. In a Monte Carlo simulation of atmospheric neutrinos [25], 95\% of the events have energies larger than about $100 \mathrm{GeV}$. The median energy is about $500 \mathrm{GeV}$. Neutrinos from a source with a $\gamma=2$ spectrum, however, are expected to carry a median energy of about $50 \mathrm{TeV}$, due to the cross-section, the muon range and the detection and event selection efficiency, which leads to a steeply rising sensitivity with energy. Table II shows relevant information on the energy distribution of Monte Carlo events, for different input spectra.

The limit setting capability of this analysis can be expressed by the sensitivity to neutrino fluxes from point sources, introduced in Section III. The sensitivity is a function of the background and describes the observation potential. In case no excess is detected over the expected background, we calculate the upper limits to the neutrino flux, as a function of both background and experimental observations. In this work we give both the sensitivity 


\begin{tabular}{c|ccc|ccc|c}
$f\left(E_{\nu}>E_{t h}\right)$ & \multicolumn{3}{c}{$\gamma=2$} & \multicolumn{3}{c}{$\gamma=3$} & Atm. \\
\hline & $\nu_{\mu}$ & $\nu_{\tau}$ & $\nu_{\mu}+\nu_{\tau}$ & $\nu_{\mu}$ & $\nu_{\tau}$ & $\nu_{\mu}+\nu_{\tau}$ & $\nu_{\mu}$ \\
$95 \%$ & 3.2 & 3.6 & 3.2 & 2.1 & 2.5 & 2.1 & 2.0 \\
$50 \%$ & 4.7 & 5.2 & 4.7 & 3.0 & 3.3 & 3.0 & 2.8 \\
$5 \%$ & 6.2 & 6.9 & 6.4 & 4.4 & 4.7 & 4.4 & 3.9
\end{tabular}

TABLE II: Energy values $\left(E_{t h}\right)$ above which a fraction of neutrino (and anti-neutrino) events $f\left(E_{\nu}>E_{t h}\right)$ is observed integrated in declination, for different input spectra. $E_{t h}$ is given in $\log _{10}\left(E_{t h} / \mathrm{GeV}\right)$. In case of atmospheric neutrinos no contribution from tau neutrinos is expected in the final data sample (see text for discussion).

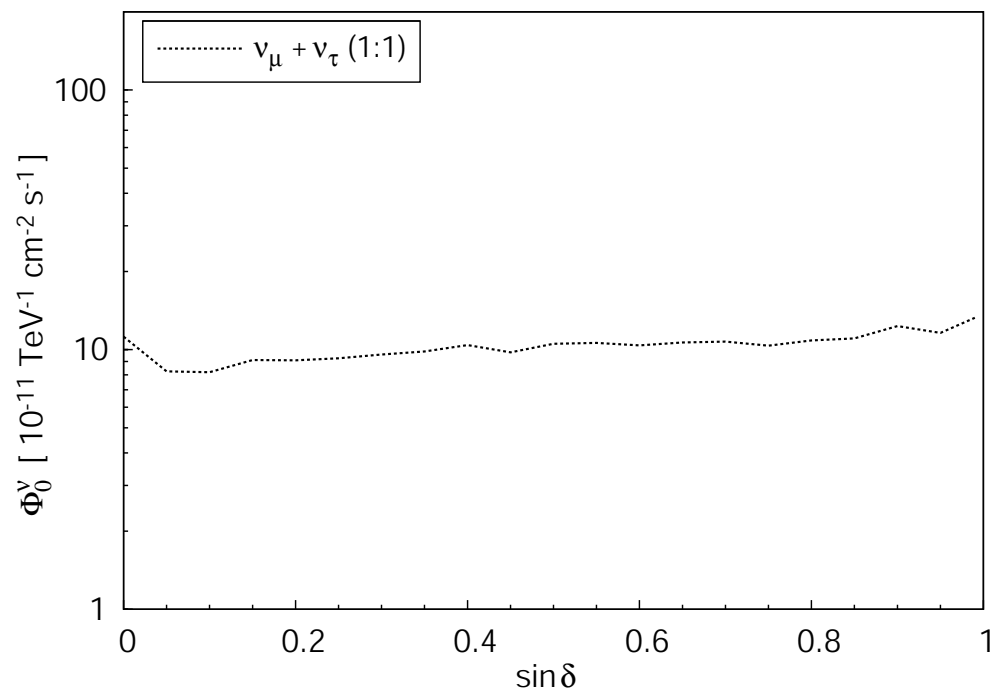

FIG. 5: Sensitivity to point sources of neutrinos as a function of declination, for an integrated exposure of 1001 days and for a $\gamma=2$ source spectrum. The sum of muon and tau neutrinos fluxes is considered assuming $\Phi_{\nu_{\mu}+\bar{\nu}_{\mu}} / \Phi_{\nu_{\tau}+\bar{\nu}_{\tau}}=1$

and upper limits to the parameter $\Phi^{0}$, i.e. on the normalization constant to the differential flux $\frac{d \Phi}{d E}=\Phi^{0} \cdot\left(\frac{\mathrm{E}}{1 \mathrm{TeV}}\right)^{-\gamma}$.

The sensitivity of this analysis to point sources of muon and tau neutrinos (and antineutrinos) with a spectral index $\gamma=2$ and averaged over declination is $\Phi_{\nu_{\mu}+\bar{\nu}_{\mu}}^{0}+\Phi_{\nu_{\tau}+\bar{\nu}_{\tau}}^{0}=$ $10.0 \cdot 10^{-11} \mathrm{TeV}^{-1} \mathrm{~cm}^{-2} \mathrm{~s}^{-1}$, assuming a flavor ratio at the earth $\Phi_{\nu_{\mu}+\bar{\nu}_{\mu}} / \Phi_{\nu_{\tau}+\bar{\nu}_{\tau}}=1$ (Figure 5 ). 


\section{SYSTEMATIC ERRORS}

\section{A. Overview}

Three main classes of systematic errors affect the searches reported in this work: the uncertainty in the optical module response (timing resolution and optical module sensitivity), the uncertainty in the modeling of the neutrino and muon propagation and interaction, and other simplifications in the simulation (propagation of photons in the ice, detector response and neutrino-muon scattering angle).

We estimate the systematic error on the rate of high energy neutrinos by variations of these quantities in the input to the simulation. The results typically depend on neutrino energy and, therefore, on the assumptions on the cosmic neutrino energy spectrum. The dominant error is due to the uncertainty in the optical module sensitivity. For a spectral index $\gamma=2$ it contributes ${ }_{-9}^{+2} \%$ of the total systematic error of ${ }_{-15}^{+10} \%$ [44].

Table 【II summarizes the systematic errors on the rate of high energy neutrinos estimated for this analysis, for three assumed energy spectra: $\gamma=2, \gamma=3$ and atmospheric energy spectrum, according to [25].

\begin{tabular}{|c|c|c|c|c|}
\hline Class & Source of uncertainty & $E^{-2}$ & $E^{-3}$ & Atm. \\
\hline 1 & $\begin{array}{l}\text { Optical module timing resolution } \\
\text { Optical module sensitivity }\end{array}$ & $\begin{array}{l} \pm 2 \% \\
+2 \% \\
-9\end{array}$ & $\begin{array}{l} \pm 2 \% \\
+5 \% \\
-17\end{array}$ & $\begin{array}{l} \pm 2 \% \\
+6 \\
{ }_{-19}^{6} \%\end{array}$ \\
\hline 2 & $\begin{array}{l}\text { Neutrino cross section and rock density } \\
\text { Muon energy loss }\end{array}$ & $\begin{array}{l} \pm 8 \% \\
\pm 1 \%\end{array}$ & $\begin{array}{l} \pm 3 \% \\
\pm 1 \%\end{array}$ & $\begin{array}{l} \pm 3 \% \\
\pm 1 \%\end{array}$ \\
\hline 3 & $\begin{array}{l}\text { Photon propagation in ice } \\
\text { Reconstruction bias } \\
\text { Neutrino-muon scattering angle }\end{array}$ & $\begin{array}{l} \pm 5 \% \\
+0 \% \\
-7 \% \\
+0 \% \\
-1\end{array}$ & $\begin{array}{l} \pm 5 \% \\
+0 \% \\
+8 \% \\
+0 \%\end{array}$ & $\begin{array}{l} \pm 5 \% \\
+0 \% \\
-9 \\
{ }_{-13}^{+0} \% \\
\end{array}$ \\
\hline & Sum & ${ }_{-15}^{+10 \%}$ & ${ }_{-21}^{+6} \%$ & ${ }_{-25}^{+7} \%$ \\
\hline
\end{tabular}

TABLE III: Summary of the systematic error in the measured rate of high energy muon neutrinos due to the three classes of systematic uncertainties, for different assumption on the energy spectrum.

An independent confirmation of the estimate of the systematic errors summarized in Table III is given by the agreement of the absolute flux prediction of atmospheric neutrinos to the experimental results. Figure 1 compares the zenith angle distribution for the observed events and the Monte Carlo simulation for atmospheric neutrinos, including the systematic error band. This confirmation is however limited by the theoretical uncertainty 
on the absolute flux prediction of atmospheric neutrinos (up to $25 \%$ or more at the highest energies [45]). The extremes of these predictions are shown in Figure 1 [25, 26], the difference representing the theoretical uncertainty due to the limited knowledge of the total primary cosmic ray flux and of the hadronic interactions in the atmosphere. It can be seen that the observed angular distribution and the event rate range between the two extremes. The theoretical uncertainty on the atmospheric neutrino flux prediction does not affect our searches for point sources, as the background is measured from the data.

The total systematic error in the neutrino rate (the sum in Table III) is asymmetric, while in the upper limit calculation errors are assumed to be Gaussian. In the limits reported in section VI we therefore round up the total error on the neutrino rate to $15 \%$ for $\gamma=2$ and $20 \%$ for $\gamma=3$. The main classes of systematic uncertainties are discussed in detail in the next sections ${ }^{2}$.

\section{B. Optical module response}

Two sources of uncertainty are related to the response of the optical modules: the timing resolution and the optical module sensitivity. The first can influence the accuracy of the track reconstruction while the second affects the effective area.

The timing of the optical modules, measured using YAG laser pulses, is better than 5 ns [1]. The impact on the rate of selected upward going events of this intrinsic resolution is less than $2 \%$, independent of the spectral index. This uncertainty is also checked by comparing the measured and the expected arrival time of photons for downward going muon tracks.

The optical module sensitivity depends on the photomultiplier quantum efficiency, on the transmission properties of the glass sphere coupled to the optical gel and on the propagation of photons in the local (re-frozen) ice surrounding the optical modules. A nominal value of the optical module sensitivity is an input parameter in the detector simulation.

A conservative overall uncertainty on the optical module sensitivity of $30 \%$ or more (including the uncertainties on the properties of the re-frozen ice) was used in previous works (e.g. [16, 33, 46]). This was dominated by the uncertainty on the optical gel transmittance

\footnotetext{
${ }^{2}$ In order to apply a symmetric systematic error on the limits a shift of $2.5 \%$ (7\%) was also applied to the signal prediction, respectively for $\gamma=2$ (3).
} 
based on laboratory measurements performed on a series of spare samples. This uncertainty can however be largely reduced by comparison of the measured and simulated zenith angle distribution of the selected upward going events. Due to the inhomogeneous distribution of the optical modules in the detector - arranged along strings in a cylindrical structure with larger vertical dimension compared to the horizontal ones - reconstructed muon tracks are differently affected by the optical module sensitivity according to their direction. We studied the effect varying the nominal value of the optical module sensitivity in the Monte Carlo simulation within a range of $\pm 30 \%$. Comparing the angular distribution of simulated and observed events, we found a $1 \sigma$ range of the best fit sensitivity corresponding to $100_{-10}^{+3} \%$ of the nominal value used in the detector simulation. The impact on the rate of high energy neutrino events is ${ }_{-9}^{+2} \%$ for $\gamma=2$ (Table III). This result is stable with respect to the other sources of systematic uncertainty. This estimate also includes the systematic error due to the uncertainty of the absolute light yield from secondary cascades along the muon track.

\section{Neutrino and muon propagation and interaction}

The number of high energy muons passing through the AMANDA-II detector for a given flux of neutrinos depends on the neutrino absorption in the earth and on the rate of neutrino interactions in the column of ice and bedrock surrounding the detector and acting as target for the production of detectable muons. Limited geophysical measurements are available to determine the rock density at the South Pole. Typical rock samples are found to vary by $10 \%$ around the nominal density of $2650 \mathrm{~kg} / \mathrm{m}^{3}$ [47] used in the Monte Carlo simulation for this analysis. This uncertainty alters the signal prediction for a neutrino flux with a spectral index of $\gamma=2$ by $2 \%$ for nearly horizontal events $\left(\delta=0^{\circ}\right.$ to $\left.30^{\circ}\right)$ and $7 \%$ for nearly vertical events $\left(\delta=60^{\circ}\right.$ to $\left.90^{\circ}\right)$. For softer spectra this uncertainty has a negligible effect. Most detectable muons are produced in the ice surrounding the detector, which has a well known density, rather than in the bedrock. This error includes the impact of this uncertainty on the muon propagation.

An uncertainty of $3 \%$ is estimated for the charged current deep-inelastic neutrino-nucleon scattering and therefore for the corresponding muon event rates. This uncertainty is estimated from the error table on the Parton Density Functions in the range between $100 \mathrm{GeV}$ and $1 \mathrm{PeV}$, as reported in [48] and the prescription for the error analysis in [49]. 
For a spectral index $\gamma=2$, these two sources of uncertainty together give an overall error of $4 \%(8 \%)$ for horizontal (vertical) events.

The rate of muons crossing the detector also depends on the muon energy loss. The processes considered in the Monte Carlo simulation for the energy loss and the production of secondaries are ionization, bremsstrahlung, pair production, photo-nuclear interaction and decay, known within an uncertainty between $1 \%$ for muon energies of about $1 \mathrm{TeV}$ and a few percent for higher energies [50]. A higher uncertainty can affect the tau energy loss at high energies [51]. However, due to the short lifetime of taus, the impact of this error on the rate of detected events is expected to be smaller than the case of muon tracks. The resulting systematic error of the absolute event rate is $1 \%$ for a variation of the cross sections by $\pm 2 \%$.

\section{Other simplifications in the simulation}

The glacial ice contains impurities which reflect past climatological changes. Calibration light sources were used to map the absorption and the scattering length of the ice and develop a wavelength and depth-dependent model of its optical properties [32]. A detailed photon propagation in the simulation is, however, computationally intensive. Therefore past AMANDA analyses used a simplified model, averaging the ice properties over the wavelengths of the Cherenkov spectrum and over the depths covered by AMANDA, optimized for the best agreement in the timing properties of observed and simulated downward going tracks. A comparison of the results for this analysis using the "average" model to more recent developments (i.e. a detailed depth-dependent simulation based on the results in [32]) gives a variation in the passing rate of the final events of $5 \%$ or less.

Residual and non-identified sources of systematic uncertainties appear when comparing the observed and the simulated cut parameter distributions at the final level, assuming that the sample is dominated by atmospheric neutrinos. A certain level of mismatch is observed for the angular resolution and the smoothness parameter. This is likely due to inaccuracies in the simulation of the detector response, e.g. non simulated cross-talk and noise hits, affecting the quality of the reconstructed tracks. A small residual fraction of misreconstructed downward going muons might also be present in the data. This is included in the background estimation and does not affect the simulation of the signal and the relative 
systematic uncertainty.

A conservative estimate of residual non-identified sources of systematic uncertainties was done by applying a scaling factor to both the smoothness and the angular resolution parameters, to obtain a good agreement between data and Monte Carlo simulation at the final level. The scaling factors necessary to obtain a good agreement in the angular resolution and in the smoothness are 1.1 and 1.07, respectively. The final event sample changes by $9 \%$ or less when using the scaled parameters rather than the unscaled ( $7 \%$ for $\gamma=2)$.

An additional systematic uncertainty is finally due to neglecting the neutrino-muon scattering angle in the simulation. In this work a neutrino induced muon is simulated as collinear to the direction of the parent neutrino, but the energy dependent average angle might exceed the size of the search bins [52] at energies below $1 \mathrm{TeV}$. For a spectral index $\gamma=2$ the systematic overestimation of the event rate when neglecting the neutrino-muon angle is less than 1\%. The tau neutrino signal prediction for this work uses a neutrino generator which properly accounts for the neutrino-muon angle [28] and is therefore free of this systematic error. Table III reports the impact of this uncertainty on the final event rates also for the case of atmospheric neutrinos, as an example of soft spectrum. This uncertainty is however not included in in Figure 1, since it only affects searches for point sources.

\section{SEARCH FOR INDIVIDUAL POINT SOURCES IN THE NORTHERN SKY}

A search for point sources of neutrinos is performed with the sample of 4282 upward going events by looking for excesses of events from the directions of known objects and by performing a survey of the full northern sky. This analysis is sensitive to a point source that would manifest itself as a statistically significant cluster of events within an angular bin consistent with the point spread function of the detector. Both surveys are based on circular search bins of a size optimized together with the event selection as described in Section III.

\section{A. Selected source candidates}

First, a sample of 32 neutrino source candidates are tested for an excess of events. The source list (Table IV) includes galactic and extragalactic objects which are identified sources 
of high energy gamma-rays located in the field of view of AMANDA-II. Any source that accelerates charged hadrons to high energies is also a possible source of detectable neutrinos from meson decay: high energy hadrons will interact with other nuclei or the ambient photon fields producing hadronic showers. In these scenarios, high energy photons and neutrinos are expected to be produced simultaneously, with correlated rates.

We consider five blazars confirmed as $\mathrm{TeV}$ gamma-ray sources [10, 12], eight blazars confirmed as (or with indications of being) GeV gamma-ray sources [13], eight galactic Xray binaries classified as micro-quasars and one neutron star binary system [3], six galactic supernova remnants and pulsars. We also consider individually selected objects, like an EGRET source with high detected flux above $100 \mathrm{MeV}$ [53], a TeV gamma-ray active galactic nucleus (M87), the bright and closest active galactic nucleus NGC 1275 and an HEGRA TeV gamma-ray source with indications of hadronic emission [54, 55].

We estimate the number of expected background events per bin $\left(n_{\mathrm{b}}\right)$ from the event density as a function of declination. Because of the rotation of the earth, any azimuthdependent variation in the event rate is averaged out and the background density is expected to be uniform in right ascension. We average the event densities over right ascension in declination bands of width comparable to the search bin radius. The statistical uncertainty in the background per search bin $\left(\sigma_{\mathrm{b}}\right)$ depends on the declination and the largest value is $7 \%$. The background estimate is not affected by systematic uncertainties, since it is independent of the Monte Carlo description of the detection efficiency.

For each search bin, the number of observed events $n_{\mathrm{obs}}$ is compared to $n_{\mathrm{b}}$. If no statistically significant excess is found, we calculate flux upper limits, which depend on the assumed source location and energy spectrum. The statistical significance of the observations is evaluated with 100 equivalent simulated experiments, using the same data sample and randomizing the events in right ascension independently for each experiment. An excess parameter $\xi$ is defined as:

$$
\xi=-\log _{10}(P)
$$

where $P$ is the binomial probability of observing $n_{\text {obs }}$ events in the search bin, given $N_{\text {band }}$ events in the declination band used for the background estimation:

$$
P\left(n_{\mathrm{obs}} \mid N_{\mathrm{band}}\right)=\left(\begin{array}{c}
N_{\mathrm{band}} \\
n_{\mathrm{obs}}
\end{array}\right) \cdot p^{n_{\mathrm{obs}}} \cdot(1-p)^{N_{\mathrm{band}}-n_{\mathrm{obs}}},
$$

where $p$ is the probability of a background event in the given search bin. The parameter 


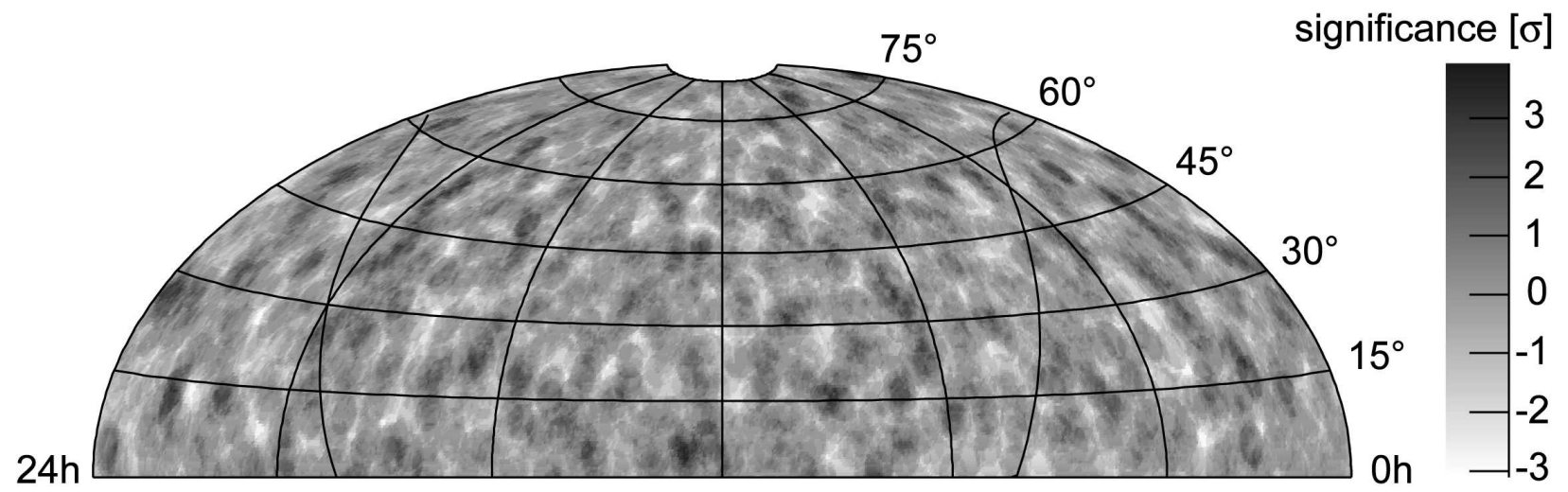

FIG. 6: Sky map of the significance obtained by scanning of the northern sky to search for event clusters. The significance is positive for excesses and negative for deficits of events compared to the expected background.

$p$ depends on the area of the search bin compared to the declination band. The distribution of the excess parameter for the source candidates agrees with the expectation for a purely atmospheric neutrino sample, simulated with the 100 samples with randomized right ascension (Figure 7).

The highest observed significance, with 8 observed events compared to 4.7 expected background events $(\xi=0.95, \sim 1.2 \sigma)$, is at the location of the $\mathrm{GeV}$ blazar $3 \mathrm{C} 273$. The second highest excess $(\xi=0.84, \sim 1.1 \sigma)$ is from the direction of the Crab Nebula, with 10 observed events compared to 6.7 expected background events.

For the directions of all selected source candidates, the observations are compatible with statistical fluctuations of the background. In Table IV we report flux upper limits for the spectral indices $\gamma=2$ and $\gamma=3$, following the Feldman and Cousins ordering principle [39] and including a Bayesian treatment of systematic errors [56, 57]. The validity range of these limits - here defined as the 90\% energy containment region - is between $1.6 \mathrm{TeV}$ and 2.5 PeV for $\gamma=2$ and between $0.1 \mathrm{TeV}$ and $25 \mathrm{TeV}$ for $\gamma=3$ (see Table II). The flux upper limits on the sum of muon and tau neutrinos are calculated under the assumption of a flavor ratio $\Phi_{\nu_{\mu}+\bar{\nu}_{\mu}} / \Phi_{\nu_{\tau}+\bar{\nu}_{\tau}}=1$ at the earth. Please note that in previous publications of the AMANDA collaboration point source limits were presented only on the flux of muon neutrinos, neglecting the sensitivity to tau neutrinos (i.e. assuming $s_{\nu_{\tau}}=0$ ). Both limit representations, the former and the current one, can be calculated and converted into each other from the values of the event upper limit $\mu_{90}$ and the expected number of signal events 


\begin{tabular}{|c|c|c|c|c|c|c|c|c|c|c|c|}
\hline \multirow[b]{2}{*}{ Candidate } & \multirow[b]{2}{*}{$\delta$} & \multirow[b]{2}{*}{$\alpha$} & \multirow[b]{2}{*}{$r$} & \multirow[b]{2}{*}{$n_{\mathrm{obs}}$} & \multirow[b]{2}{*}{$n_{\mathrm{b}}$} & \multicolumn{2}{|l|}{$\gamma=2$} & \multicolumn{4}{|c|}{$\gamma=3$} \\
\hline & & & & & & $\overbrace{\mu_{90} s_{\nu_{\mu}} s_{\nu_{\tau}}}$ & $\overline{\Phi_{\nu_{\mu}}^{0}+\Phi_{\nu_{\tau}}^{0}}$ & $\mu_{90}$ & $s_{\nu_{\mu}}$ & $\widehat{s}_{\nu_{\tau}}$ & $\overline{\Phi_{\nu_{\mu}}^{0}+\Phi_{\nu_{\tau}}^{0}}$ \\
\hline \multicolumn{12}{|c|}{ TeV blazars } \\
\hline Markarian 421 & 38.2 & 11.1 & 3.25 & 6 & 7.4 & $\begin{array}{llll}4.1 & 0.97 & 0.15\end{array}$ & 7.4 & 4.1 & 0.15 & 0.01 & 51 \\
\hline Markarian 501 & 39.8 & 16.9 & 3.00 & 8 & 6.4 & 7.90 .930 .14 & 14.7 & 8.3 & 0.15 & 0.01 & 102 \\
\hline 1ES $1426+428$ & 42.7 & 14.5 & 2.75 & 5 & 5.5 & 4.80 .900 .13 & 9.4 & 4.8 & 0.16 & 0.01 & 58 \\
\hline 1ES $2344+514$ & 51.7 & 23.8 & 2.50 & 4 & 6.2 & 3.10 .890 .15 & 5.9 & 3.1 & 0.19 & 0.01 & 29 \\
\hline 1ES 1959+650 & 65.1 & 20.0 & 2.25 & 5 & 4.8 & $\begin{array}{lll}5.6 & 0.71 & 0.11\end{array}$ & 13.5 & 5.6 & 0.21 & 0.02 & 48 \\
\hline \multicolumn{12}{|c|}{ GeV blazars } \\
\hline $3 \mathrm{C} 273$ & 2.1 & 12.5 & 3.75 & 8 & 4.7 & $\begin{array}{llll}9.6 & 0.96 & 0.10\end{array}$ & 18.0 & 9.8 & 0.04 & $\sim 0$ & 427 \\
\hline QSO $0528+134$ & 13.4 & 5.5 & 3.50 & 4 & 6.1 & 3.21 .060 .14 & 5.3 & 3.2 & 0.08 & 0.01 & 72 \\
\hline QSO $0235+164$ & 16.6 & 2.6 & 3.50 & 7 & 6.1 & 6.71 .030 .14 & 11.4 & 7.1 & 0.09 & 0.01 & 145 \\
\hline QSO $1611+343$ & 34.4 & 16.2 & 3.25 & 6 & 7.0 & $\begin{array}{lll}4.5 & 0.95 & 0.15\end{array}$ & 8.3 & 4.8 & 0.14 & 0.01 & 65 \\
\hline QSO $1633+382$ & 38.2 & 16.6 & 3.25 & 9 & 7.4 & 8.10 .970 .15 & 14.6 & 8.3 & 0.15 & 0.01 & 103 \\
\hline QSO $0219+428$ & 42.9 & 2.4 & 2.75 & 5 & 5.5 & $\begin{array}{lll}4.9 & 0.89 & 0.13\end{array}$ & 9.6 & 4.8 & 0.16 & 0.01 & 58 \\
\hline QSO $0954+556$ & 55.0 & 9.9 & 2.50 & 2 & 6.7 & 1.40 .910 .15 & 2.7 & 1.4 & 0.20 & 0.01 & 12 \\
\hline QSO $0716+714$ & 71.3 & 7.4 & 2.25 & 1 & 4.0 & 1.20 .700 .13 & 3.0 & 1.2 & 0.20 & 0.02 & 11 \\
\hline \multicolumn{12}{|c|}{ Other $A G N s$} \\
\hline M 87 & 12.4 & 12.5 & 3.50 & 6 & 6.1 & 5.31 .070 .14 & 8.7 & 5.7 & 0.08 & 0.01 & 134 \\
\hline NGC 1275 & 41.5 & 3.3 & 3.00 & 4 & 6.8 & 2.70 .950 .14 & 5.0 & 2.8 & 0.16 & 0.01 & 31 \\
\hline \multicolumn{12}{|c|}{ Micro-quasars and neutron star binaries } \\
\hline SS433 & 5.0 & 19.2 & 3.75 & 4 & 6.1 & 3.11 .160 .13 & 4.8 & 3.1 & 0.06 & $\sim 0$ & 96 \\
\hline GRS $1915+105$ & 10.9 & 19.3 & 3.50 & 7 & 6.1 & 6.81 .080 .14 & 11.2 & 7.1 & 0.07 & $\sim 0$ & 184 \\
\hline $\mathrm{AO} 0535+26$ & 26.3 & 5.7 & 3.50 & 7 & 6.5 & 6.40 .990 .14 & 11.3 & 6.7 & 0.11 & 0.01 & 112 \\
\hline GRO J0422+32 & 32.9 & 4.4 & 3.25 & 9 & 6.7 & 9.00 .940 .14 & 16.7 & 9.0 & 0.14 & 0.01 & 123 \\
\hline Cygnus X-1 & 35.2 & 20.0 & 3.25 & 8 & 7.0 & 7.30 .950 .15 & 13.2 & 7.3 & 0.14 & 0.01 & 96 \\
\hline Cygnus X-3 & 41.0 & 20.5 & 3.00 & 7 & 6.5 & 6.40 .950 .14 & 11.8 & 6.8 & 0.16 & 0.01 & 80 \\
\hline XTE J1118+480 & 48.0 & 11.3 & 2.75 & 3 & 7.1 & $\begin{array}{lll}1.5 & 0.97 & 0.14\end{array}$ & 2.8 & 1.5 & 0.19 & 0.01 & 15 \\
\hline CI Cam & 56.0 & 4.3 & 2.50 & 9 & 6.3 & 9.40 .910 .14 & 17.8 & 9.5 & 0.20 & 0.01 & 88 \\
\hline LS I +61303 & 61.2 & 2.7 & 2.25 & 5 & 4.8 & $\begin{array}{lll}5.6 & 0.75 & 0.13 \\
\end{array}$ & 12.6 & 5.6 & 0.20 & 0.01 & 50 \\
\hline \multicolumn{12}{|c|}{ SNR and pulsars } \\
\hline$\overline{\text { SGR } 1900+14}$ & 9.3 & 19.1 & 3.50 & 5 & 5.7 & 4.81 .090 .13 & 7.8 & 4.8 & 0.07 & $\sim 0$ & 127 \\
\hline Geminga & 17.9 & 6.6 & 3.50 & 3 & 6.2 & 2.01 .010 .14 & 3.5 & 2.0 & 0.10 & 0.01 & 38 \\
\hline Crab Nebula & 22.0 & 5.6 & 3.50 & 10 & 6.7 & 10.10 .980 .15 & 17.8 & 10.4 & 0.10 & 0.01 & 192 \\
\hline PSR $1951+32$ & 32.9 & 19.9 & 3.25 & 4 & 6.7 & 2.70 .940 .14 & 5.0 & 2.7 & 0.14 & 0.01 & 38 \\
\hline Cassiopeia A & 58.8 & 23.4 & 2.50 & 5 & 6.0 & 4.40 .860 .13 & 8.9 & 4.4 & 0.20 & 0.01 & 41 \\
\hline PSR J0205+6449 & 64.8 & 2.1 & 2.25 & 1 & 4.7 & 1.30 .720 .11 & 3.1 & 1.3 & 0.21 & 0.02 & 11 \\
\hline \multicolumn{12}{|c|}{ Unidentified high energy gamma-ray sources } \\
\hline $3 \mathrm{EG}$ J0450+1105 & 11.4 & 4. & 3.50 & 8 & 5.9 & 8.41 .080 .14 & 13.8 & 8.6 & 0.08 & $\sim 0$ & 218 \\
\hline TeV J2032+4131 & 41.5 & 20.5 & 3.00 & 7 & 6.8 & $\begin{array}{lll}6.1 & 0.95 & 0.14\end{array}$ & 11.2 & 6.5 & 0.16 & 0.01 & 76 \\
\hline
\end{tabular}

TABLE IV: Flux upper limits for selected neutrino source candidates: source directions (declination $\delta$ in degrees and right ascension $\alpha$ in hours), search bin size ( $r$ in degrees), number of observed events $\left(n_{\mathrm{obs}}\right)$ and expected background $\left(n_{\mathrm{b}}\right) . \mu_{90}$ is the event upper limit at $90 \%$ CL (different for $\gamma=2$ and $\gamma=3$ because of different systematic errors) and $s_{\nu_{\mu}}\left(s_{\nu_{\tau}}\right)$ is the expected number of events from muon (tau) neutrino and anti-neutrino interactions for a differential flux $\frac{\mathrm{d} \Phi}{\mathrm{dE}}=10^{-11} \cdot\left(\frac{\mathrm{E}}{1 \mathrm{TeV}}\right)^{-\gamma}$ $\mathrm{TeV}^{-1} \mathrm{~cm}^{-2} \mathrm{~s}^{-1}$. The $90 \% \mathrm{CL}$ upper limits $\left(\Phi_{\nu_{\mu}}^{0}+\Phi_{\nu_{\tau}}^{0}\right.$, neutrino and anti-neutrinos) are given in units of $10^{-11} \mathrm{TeV}^{-1} \mathrm{~cm}^{-2} \mathrm{~s}^{-1}$, for both spectral indices $\gamma=2$ and $\gamma=3$. 


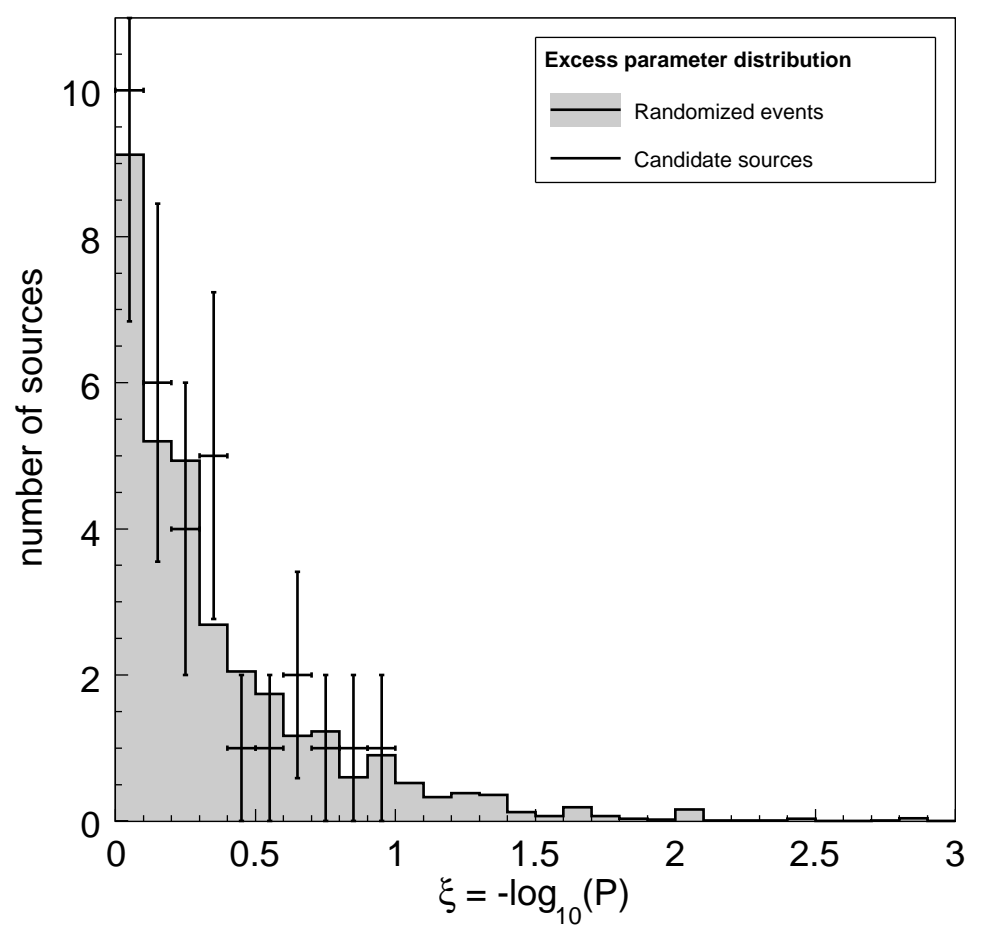

FIG. 7: Excess parameter $(\xi)$ distribution from the search for neutrinos from pre-selected objects. The results of each individual observation (data points) are compared to the expected distribution from 1000 simulated equivalent experiments with randomized right ascension (filled histogram).

from muon (tau) neutrinos and antineutrinos $s_{\nu_{\mu}}\left(s_{\nu_{\tau}}\right)$ given in Table IV:

$$
\Phi_{\nu_{\mu}+\bar{\nu}_{\mu}}^{0}=\frac{1}{2}\left(\Phi_{\nu_{\mu}+\bar{\nu}_{\mu}}^{0}+\Phi_{\nu_{\tau}+\bar{\nu}_{\tau}}^{0}\right)=\frac{\mu_{90}}{s_{\nu_{\mu}}+s_{\nu_{\tau}}} 10^{-11} \mathrm{TeV}^{-1} \mathrm{~cm}^{-2} \mathrm{~s}^{-1}
$$

All limits include a systematic uncertainty of $15 \%$ (20\%) in the signal prediction for $\gamma=2(3)$ (see Section V) and a statistical error of $7 \%$ in the background estimation.

\section{B. Northern sky survey}

A full scan of the northern sky is also performed to look for any localized event cluster, using a grid of circular search sky bins centered at distances of $0.5^{\circ}$. The bin size is chosen according to the optimization results reported in Section [II]. The strong bin correlation in this method ensures a high detection chance without a high trial factor penalty and does not require grid-shifts to account for boundary leaking effects [58].

The background per bin is estimated in the same way as in the search for neutrinos from 


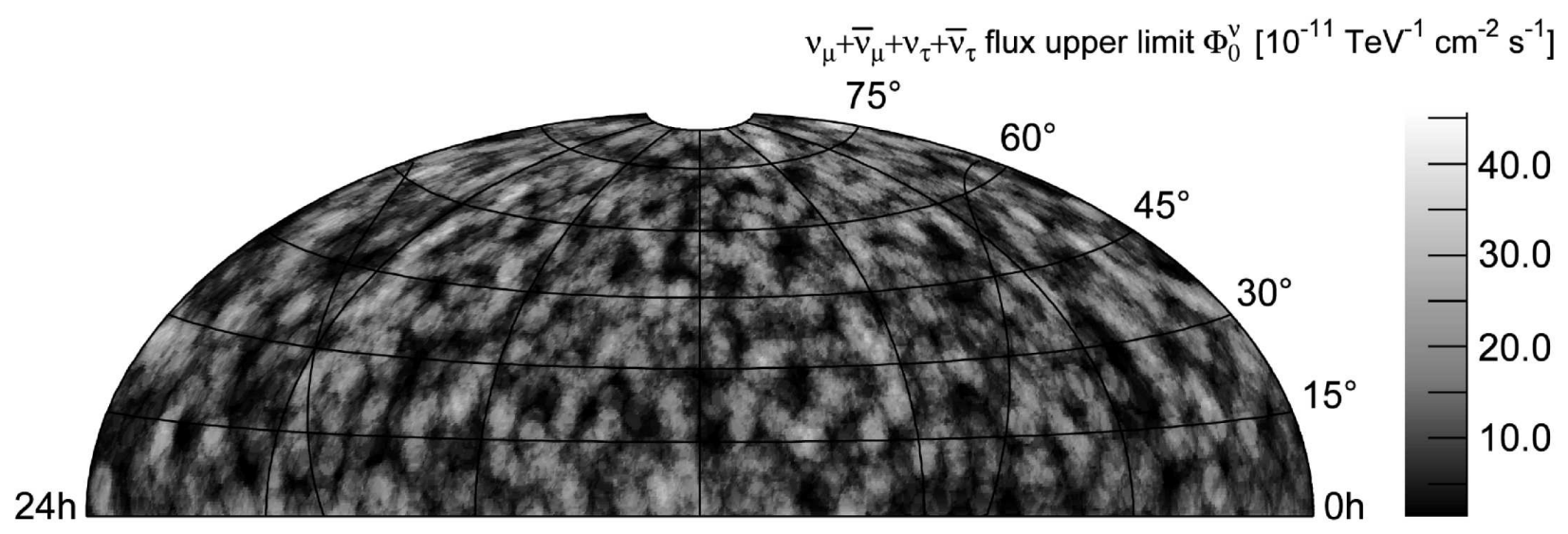

FIG. 8: Map of the $90 \%$ CL upper limits to the muon and tau neutrino flux from the survey of the northern sky, for a spectral index $\gamma=2$. Limits are given on the normalization factor $\Phi^{0}$ to the flux $\frac{\mathrm{d} \Phi}{\mathrm{dE}}=\Phi^{0} \cdot\left(\frac{\mathrm{E}}{1 \mathrm{TeV}}\right)^{-\gamma}$.

known candidates, but a singularity arises at the pole, where this method is not reliable. We therefore limited this search to events with declination below $85^{\circ}$, giving 4251 remaining upgoing muons. The statistical significance of any deviation is evaluated with the same technique as above. Simulations with randomized events are performed using the same grid of search bins, to account for the trial factor and the bin-to-bin correlations.

Figure [6 shows the distribution of observed significances, in standard deviations. All observed excesses and deficits are compatible with statistical fluctuations of background. The highest positive deviation corresponds to about $3.7 \sigma$. The probability of such a deviation or higher due to background, estimated with 100 equivalent sky surveys of events with randomized right ascension, is $69 \%$.

Also in this case we give the neutrino flux upper limits as a function of declination and right ascension including the systematic uncertainty discussed in Section $\nabla$. The results for a spectral index $\gamma=2$ are shown in Fig. 8. The experimentally observed upper limit for $\gamma=2$ (averaged over declination and right ascension) is $\Phi_{\nu_{\mu}+\bar{\nu}_{\mu}}^{0}+\Phi_{\nu_{\tau}+\bar{\nu}_{\tau}}^{0}=10.6 \cdot 10^{-11} \mathrm{TeV}^{-1} \mathrm{~cm}^{-2} \mathrm{~s}^{-1}$, without systematic error and $\Phi_{\nu_{\mu}+\bar{\nu}_{\mu}}^{0}+\Phi_{\nu_{\tau}+\bar{\nu}_{\tau}}^{0}=11.1 \cdot 10^{-11} \mathrm{TeV}^{-1} \mathrm{~cm}^{-2} \mathrm{~s}^{-1}$ with systematic error. The first can be compared to the sensitivity $\left(\Phi_{\nu_{\mu}+\bar{\nu}_{\mu}}^{0}+\Phi_{\nu_{\tau}+\bar{\nu}_{\tau}}^{0}=10.0\right.$. $10^{-11} \mathrm{TeV}^{-1} \mathrm{~cm}^{-2} \mathrm{~s}^{-1}$ ), which gives the expected average upper limit before the experimental observations are performed. The agreement between the two quantities is an independent confirmation of the compatibility of the experimental observations with the estimated back- 
ground.

The results of this sky survey are compared to those from an analysis using an independent cluster search algorithm that is based on an un-binned likelihood procedure in which the events are weighted with the individual track angular resolution [59]. The two methods yield consistent results for the significance map.

\section{SEARCH FOR SPATIALLY CORRELATED AND CUMULATIVE EXCESSES}

As no statistically significant accumulation of events could be established in the sky map, two searches for cumulative effects are also made. The first search aims at detecting correlations between spatial event coordinates, independent of source candidates; the second tests the cumulative significance of pre-defined catalogs of objects (source stacking). The purpose is to search for an excess of events from the sum of several sources, where the individual fluxes are below the detector sensitivity but the integrated signal yields a significant excess over the background.

\section{A. Search for an excess at small event separation angles}

The combined effect from several weak sources can be observed as an excess of event pairs at a small angular distance, consistent with the point spread function of the detector. The number of individual sources and their location would remain undefined.

We look for angular correlations in the final event sample by comparing the distribution of the squared separation angle of event pairs to a template distribution expected for atmospheric neutrinos. The latter is obtained from 10000 neutrino samples randomizing the right ascension of the 4282 observed events. This properly takes into account the expected declination distribution, which is not uniform because of the effective area and the angular distribution of primary cosmic rays ${ }^{3}$.

The distribution of the separation angle between event pairs is shown in Figure 9, with the corresponding $1 \sigma$ confidence belt. The potential to identify a signal contribution with

\footnotetext{
${ }^{3}$ Please note that this randomization technique implies that the statistical test described below is insensitive to potential sources at declinations higher than $\delta \approx 85^{\circ}$.
} 


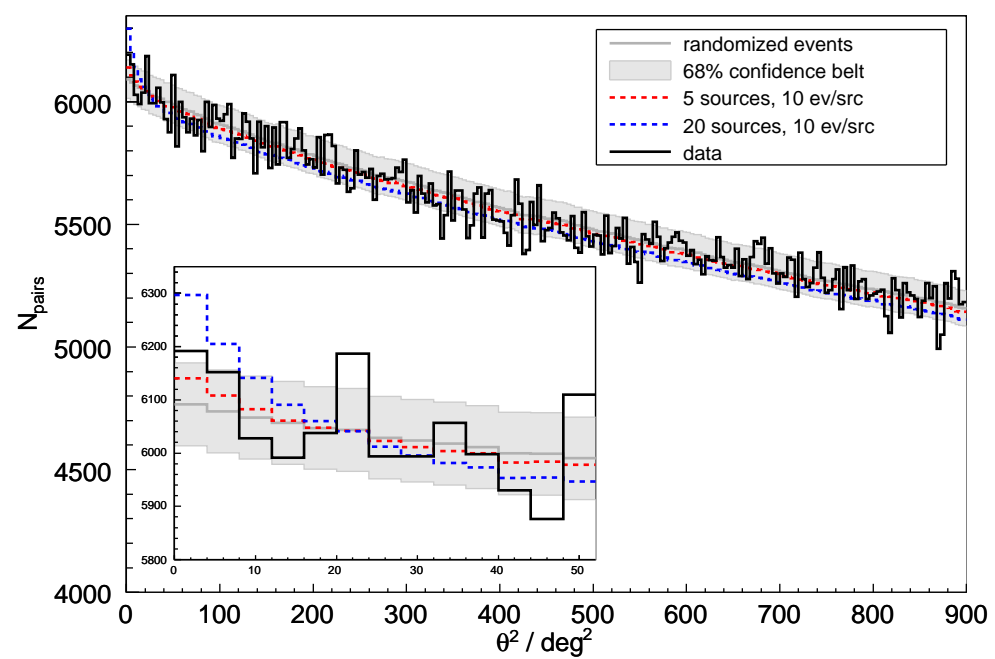

FIG. 9: Distribution of the separation angle between event pairs for the experimental data and for two samples consisting of the original data plus signal events following the point spread function of the detector (5 and 20 sources respectively, each contributing 10 neutrinos on average). The distribution expected for a pure atmospheric sample is also shown (randomized events) with a $1 \sigma$ confidence belt.

this method is also shown for the cases of 5 and 20 sources respectively, each contributing on average 10 neutrinos to the final event sample. An accumulation at small separation angles would be expected.

In order to detect a signal at a $3 \sigma$ level, a reduced $\chi^{2}$ value of more than 1.31 should be found in the comparison of the data with the template. The reduced $\chi^{2}$ value obtained with the observed event sample is $203 / 223$ for 223 degrees of freedom and the probability to obtain a larger or equal value is $81 \%$. No indications of a contribution from an ensemble of weak neutrino sources is therefore found by this test. The probability of a $3 \sigma$ detection would be $11 \%$ and $65 \%$ for 5 and 20 sources, respectively.

In an alternative approach the sky-map is tested for correlated events and distortions of the angular distribution resulting from many weak sources. The sky-map is decomposed by means of spherical harmonics and the power spectrum of their coefficients is analyzed. This study will be presented elsewhere. 


\section{B. Source stacking results}

The final data set has been searched for a signal due to the cumulative neutrino flux from generic classes of active galactic nuclei. Recently, a source stacking analysis was performed on data collected in the year 2000 [34, 60], defining samples of 10 classes of active galactic nuclei, according to phenomenological selection criteria. The event sample extracted in this work yields an increase in sensitivity by a factor 4 compared to [34, 60].

The number of sources in each class was optimized assuming a linear correlation between the photon and the neutrino flux. The size of circular search bins was optimized according to the point spread function of this analysis.

For each source class sample, the cumulative signal and the background expectation are determined as the sum of the corresponding quantities of the individual sources. To ensure a statistically correct treatment of overlapping search bins, events in the overlap area contribute only once to the cumulative signal. The background estimation is also corrected for the bin correlations.

None of the classes tested show a statistically significant excess over the background expectations. Table $\mathrm{V}$ reports the $90 \%$ confidence level upper limits to the cumulative neutrino flux, following the Feldman and Cousins ordering principle [39] and including a Bayesian treatment of systematic errors [56, 57]. The limits include a systematic error of $15 \%$ in the signal prediction (see Section $\nabla$ ) and a statistical error in the background estimation between $3 \%$ and $7 \%$, depending on declination (Section VI). The limits do not include the contribution from tau neutrinos.

\section{SUMMARY}

We have performed a search for point sources of high energy neutrinos in the northern sky with the data collected with the AMANDA-II telescope in the years 2000 to 2004. Improved event reconstruction and selection techniques have been applied, with special emphasis on the energy spectrum of the Monte Carlo events passing the selection cuts and aiming at good sensitivity to a large variety of possible signal energy spectra.

We selected the largest event sample ever extracted from data collected with a neutrino telescope, consisting of 4282 upward going muon tracks with good reconstruction qual- 


\begin{tabular}{r|cccccc} 
AGN class & $N_{\mathrm{src}}$ & $n_{\mathrm{obs}}$ & $n_{\mathrm{b}}$ & $\mu_{90}$ & $\Phi_{\nu_{\mu}}^{0}$ & $\Phi_{\nu_{\mu}}^{0} / N_{\mathrm{src}}$ \\
\hline GeV blazars & 8 & 17 & 25.7 & 2.7 & 2.7 & 0.34 \\
unidentified GeV sources & 22 & 75 & 77.5 & 14.1 & 16.5 & 0.75 \\
IR blazars & 11 & 40 & 43.0 & 9.3 & 10.6 & 0.96 \\
keV blazars (HEAO-A) & 3 & 9 & 14.0 & 2.7 & 3.6 & 1.18 \\
keV blazars (ROSAT) & 8 & 31 & 33.4 & 8.3 & 9.7 & 1.20 \\
TeV blazars & 5 & 19 & 23.6 & 4.7 & 5.5 & 1.11 \\
GPS and CSS & 8 & 24 & 29.5 & 5.0 & 5.9 & 0.74 \\
FR-I galaxies & 1 & 3 & 3.1 & 4.3 & 4.1 & 4.11 \\
FR-I without M87 & 17 & 40 & 57.2 & 2.7 & 2.9 & 0.17 \\
FR-II galaxies & 17 & 77 & 68.5 & 25.5 & 30.4 & 1.79 \\
radio-weak quasars & 11 & 35 & 41.6 & 5.6 & 6.7 & 0.61
\end{tabular}

TABLE V: Results of the stacking analysis for each AGN class: number of sources $\left(N_{\text {src }}\right)$, number of expected background events $\left(n_{\mathrm{b}}\right)$ and number of observed events $\left(n_{\mathrm{obs}}\right) . \mu_{90}$ is the cumulative event upper limit and $\Phi_{\nu_{\mu}}^{0}$ is the upper limit to the cumulative muon flux, in units of $10^{-11} \mathrm{TeV}^{-1} \mathrm{~cm}^{-2} \mathrm{~s}^{-1}$, for a spectral index $\gamma=2$. The last column gives the limits divided by the number of sources $\left(\Phi_{\nu_{\mu}}^{0} / N_{\text {src }}\right)$. These limits do not include the contribution of tau neutrinos.

ity. This is in agreement with a Monte Carlo simulation of atmospheric neutrinos yielding $4600_{-1000}^{+300}$ (sys) events (Figure 1). The effects of oscillations of atmospheric neutrinos, yielding muon neutrino disappearance and tau neutrino appearance are negligible and the contribution of tau neutrinos to this event sample is below $0.5 \%$.

In contrast to that, we emphasize that in case of cosmic neutrinos a contribution from tau neutrinos is expected in the sample of up-going muon tracks selected in this analysis, depending on declination and energy spectrum. We therefore also estimated the contribution from charged current interactions of tau neutrinos followed by tau decay into a muon (with a $17.7 \%$ branching ratio). Under the assumption $\Phi_{\nu_{\mu}+\bar{\nu}_{\mu}} / \Phi_{\nu_{\tau}+\bar{\nu}_{\tau}}=1$ at the earth, the additional contribution to the event sample from tau neutrinos, ranges from $10 \%$ to $16 \%$ for $\gamma=2$, depending on declination.

The sensitivity to a point source flux of muon and tau neutrinos (and anti-neutrinos) is $\Phi_{\nu_{\mu}+\bar{\nu}_{\mu}}^{0}+\Phi_{\nu_{\tau}+\bar{\nu}_{\tau}}^{0}=10.0 \cdot 10^{-11} \mathrm{TeV}^{-1} \mathrm{~cm}^{-2} \mathrm{~s}^{-1}$, for 1001 effective days of exposure, in the energy range between $1.6 \mathrm{TeV}$ and $2.5 \mathrm{PeV}$ and assuming a flavor ratio at earth of $\Phi_{\nu_{\mu}+\bar{\nu}_{\mu}}^{0} / \Phi_{\nu_{\tau}+\bar{\nu}_{\tau}}^{0}=1$. This is the declination-averaged sensitivity on the normalization factor $\Phi^{0}$ to the flux $\frac{\mathrm{d} \Phi}{\mathrm{dE}}=\Phi^{0} \cdot\left(\frac{\mathrm{E}}{1 \mathrm{TeV}}\right)^{-\gamma}$, assuming $\gamma=2$. The representation is different from our previous papers, where the sensitivity was given to the integrated muon neutrino flux and 
additionally the contribution from tau neutrinos was neglected. For this work the sensitivity to the muon neutrino component of the flux would be $\Phi_{\nu_{\mu}+\bar{\nu}_{\mu}}^{0}=1 / 2\left(\Phi_{\nu_{\mu}+\bar{\nu}_{\mu}}^{0}+\Phi_{\nu_{\tau}+\bar{\nu}_{\tau}}^{0}\right)=$ $5.0 \cdot 10^{-11} \mathrm{TeV}^{-1} \mathrm{~cm}^{-2} \mathrm{~s}^{-1}$.

The average experimentally observed upper limit of this analysis for $\gamma=2$, averaged over declination and right ascension, is $\Phi_{\nu_{\mu}+\bar{\nu}_{\mu}}^{0}+\Phi_{\nu_{\tau}+\bar{\nu}_{\tau}}^{0}=10.6 \cdot 10^{-11} \mathrm{TeV}^{-1} \mathrm{~cm}^{-2} \mathrm{~s}^{-1}$, without systematic error and $\Phi_{\nu_{\mu}+\bar{\nu}_{\mu}}^{0}+\Phi_{\nu_{\tau}+\bar{\nu}_{\tau}}^{0}=11.1 \cdot 10^{-11} \mathrm{TeV}^{-1} \mathrm{~cm}^{-2} \mathrm{~s}^{-1}$, including the systematic error, consistent with the expected sensitivity. An overall improvement of approximately four (1.5) times is achieved compared to the sensitivity after 197 (607) days of exposure [34, 35], when neglecting the sensitivity to tau neutrinos in this comparison. The improvement is due to the longer live-time and a refined event selection yielding a higher signal efficiency and a better background rejection power.

We searched the sample of 4282 up-going muon tracks for a signal of cosmic origin, testing first individual directions. Table IV reports the results of the searches on a catalog of 32 selected sources. The highest excess, with a pre-trial significance of $1.2 \sigma$, corresponds to the direction of the blazar 3C 273. The highest excess from the full northern sky corresponds to a pre-trial significance of $3.7 \sigma$ (Figure 6) ). The probability of such a deviation or higher due to background is $69 \%$. We also performed a search based on the angular separation of the events (Figure 9) and a stacking analysis of selected active galactic nuclei (Table V).

No indication of point sources of neutrinos was found. Therefore we present flux upper limits for the 32 source candidates, assuming spectral indices $\gamma=2$ and $\gamma=3$, at a $90 \%$ confidence level (Table IV). Additionally we provide a map of the flux upper limit for $\gamma=2$ for the full northern sky. We give the most stringent flux upper limits to date.

\section{ACKNOWLEDGMENTS}

We acknowledge the support from the following agencies: National Science FoundationOffice of Polar Program, National Science Foundation-Physics Division, University of Wisconsin Alumni Research Foundation, Department of Energy, and National Energy Research Scientific Computing Center (supported by the Office of Energy Research of the Department of Energy), the NSF-supported TeraGrid system at the San Diego Supercomputer Center (SDSC), and the National Center for Supercomputing Applications (NCSA); Swedish Research Council, Swedish Polar Research Secretariat, and Knut and Alice Wallenberg Foun- 
dation, Sweden; German Ministry for Education and Research, Deutsche Forschungsgemeinschaft (DFG), Germany; Fund for Scientific Research (FNRS-FWO), Flanders Institute to encourage scientific and technological research in industry (IWT), Belgian Federal Office for Scientific, Technical and Cultural affairs (OSTC); the Netherlands Organisation for Scientific Research (NWO); M. Ribordy acknowledges the support of the SNF (Switzerland); J. D. Zornoza acknowledges the Marie Curie OIF Program (contract 007921).

[1] E. Andrés et al. (The AMANDA Collaboration), Astropart. Phys. 13, 1 (2000).

[2] B. Link and F. Burgio, Phys. Rev. Lett. 94, 181101 (2005).

[3] L. Anchordoqui et al., Astrophys. J. 589, 481 (2003).

[4] F. Aharonian et al., J. Phys. Conf. 39, 408 (2006).

[5] D. Torres and F. Halzen (2006), submitted to Astron. Astrophys., astro-ph/0607368.

[6] C. Distefano et al., Astrophys. J. 575, 378 (2002).

[7] W. Bednarek et al., New Astron. Rev. 49, 1 (2005).

[8] F. Stecker, Phys. Rev. D 72, 107301 (2005).

[9] J. Alvarez-Muniz and P. Meszaros, Phys. Rev. D 70, 123001 (2004).

[10] K. Mannheim, Phys. Rev. D 63, 023003 (2001).

[11] A. Muecke and R. J. Protheroe, Astropart. Phys. 15, 121 (2001).

[12] A. Muecke et al., Astropart. Phys. 18, 593 (2003).

[13] A. Neronov and D. Semikoz, Phys. Rev. D 66, 123003 (2002).

[14] J. Learned and K. Mannheim, Ann. Rev. Nucl. Part. Sci. 50, 679 (2000).

[15] J. Ahrens et al. (The AMANDA Collaboration), Phys. Rev. Lett. 90, 251101 (2003).

[16] M. Ackermann et al. (The AMANDA Collaboration), Astropart. Phys. 22, 127 (2004).

[17] V. Aynutdinov et al., Astropart. Phys. 25, 140 (2006).

[18] W. Rhode et al., Astropart. Phys. 4, 217 (1996).

[19] M. Ambrosio et al., Astropart. Phys. 19, 1 (2003).

[20] K. Abe et al. (2006), submitted.

[21] M. Ambrosio et al., Astrophys. J. 546, 1038 (2001).

[22] R. Svoboda et al., Astrophys. J. 444, 415 (1995).

[23] H. Adarkar et al., Astrophys. J. 380, 235 (1993). 
[24] Y. Oyama et al., Phys. Rev. D 39, 1481 (1989).

[25] P. Lipari, Astropart. Phys. 1, 195 (1993).

[26] M. Honda et al., Phys. Rev. D 70, 043008 (2004).

[27] J. Ahrens et al. (The AMANDA Collaboration), Nucl. Instrum. Meth. A 524, 169 (2004).

[28] A. Gazizov and M. Kowalski, Comput. Phys. Commun. 172, 203 (2005).

[29] K. Hagiwara et al., Phys. Rev. D 66, 010001 (2002).

[30] T. Gaisser et al., Phys. Rept. 258, 173 (1995).

[31] F. Halzen and D. Hooper, Rept. Prog. Phys. 65, 1025 (2002).

[32] M. Ackermann et al. (The AMANDA Collaboration), J. Geophys. Res. 111, D13203 (2006).

[33] J. Ahrens et al. (The AMANDA Collaboration), Astrophys. J. 583, 1040 (2003).

[34] J. Ahrens et al. (The AMANDA Collaboration), Phys. Rev. Lett. 92, 071102 (2004).

[35] M. Ackermann et al. (The AMANDA Collaboration), Phys. Rev. D 71, 077102 (2005).

[36] A. Pohl, Licentiate thesis, Uppsala Universitet (2004).

[37] T. Neunhöffer, Astropart. Phys. 25, 220 (2006).

[38] G. Hill and K. Rawlins, Astropart. Phys. 19, 393 (2003).

[39] G. Feldman and R. Cousins, Phys. Rev. D 57, 3873 (1998).

[40] J. Ahrens et al. (The AMANDA Collaboration), Phys. Rev. D 66, 012005 (2002).

[41] S. Eidelman et al., Phys. Lett. B B592, 1 (2004), http://pdg.lbl.gov.

[42] T. Kashti and E. Waxman, Phys. Rev. Lett. 95, 181101 (2005).

[43] Y. Ashie et al., Phys. Rev. D 71, 112005 (2005).

[44] M. Ackermann, Ph.D. thesis, Humboldt-Universität, Berlin (2006).

[45] T. Gaisser and M. Honda, Ann. Rev. Nucl. Part. Sci. 52, 153 (2002).

[46] J. Ahrens et al. (The AMANDA Collaboration), Phys. Rev. D 67, 012003 (2003).

[47] W. Lowry, Fundamentals of Geophysics (Cambridge University Press, 1997).

[48] E. Berger et al. (The CTEQ Collaboration) (2005), uRL, www.phys.psu.edu/ cteq/.

[49] J. Pumplin et al., Journal of High Energy Physics 07 (2002).

[50] E. Bugaev, I. Sokalski, and S. Klimushin (2000), hep-ph/0010323.

[51] E. Bugaev et al., Astropart. Phys. 21, 491 (2004).

[52] T. Gaisser, Cosmic rays and Particle physics (Cambridge University Press, 1990).

[53] R. Hartman et al., Astrophys. J. Suppl. Ser. 123, 79 (1999).

[54] F. Aharonian et al., Astron. Astrophys. 370, 112 (2001). 
[55] Y. Butt et al., Astrophys. J. 497, 494 (2003).

[56] G. Hill, Phys. Rev. D 67, 118101 (2003).

[57] J. Conrad et al., Phys. Rev. D 67, 012002 (2003).

[58] T. Hauschildt, Ph.D. thesis, Humboldt-Universität, Berlin (2004).

[59] T. Neunhöffer and L. Köpke, Nucl. Instrum. Meth. A 558, 561 (2006).

[60] A. Achterberg et al. (The IceCube Collaboration) (2006), On the selection of AGN neutrino source candidates for a source stacking analysis with neutrino telescopes, accepted by Astropart. Phys. 